\title{
UV-induced tetrazole-thiol reaction for polymer conjugation and surface functionalization
}

\author{
Wenqian Feng, Linxian Li, Chengwu Yang, Alexander Welle, Oliver Trapp and Pavel A. Levkin*
}

Abstract: A UV-induced 1,3-dipolar nucleophilic addition of tetrazoles to thiols is described. Under UV irradiation the reaction proceeds rapidly at room temperature, with high yields, without a catalyst, and in both polar protic and aprotic solvents, including water. This UV-induced tetrazole-thiol reaction was successfully applied for synthesis of small molecules, protein modification, and rapid and facile polymer-polymer conjugation. The reaction has been also demonstrated for the formation of micropatterns by site-selective surface functionalization. Superhydrophobic-hydrophilic micropatterns were successfully created by sequential modifications of a tetrazole-modified porous polymer surface with hydrophobic and hydrophilic thiols. A biotin functionalized surface could be fabricated in aqueous solutions under long-wavelength UV irradiation.

Ever since the first reported photoreaction of an organic compound, santonin, in 1834 by Trommsdorf, ${ }^{[1]}$ the spatially and temporally controllable photochemistry has found diverse and widespread applications, ${ }^{[2]}$ including surface functionalization to create patterned or gradient immobilization of various substrates. ${ }^{[3]}$ Photo-induced click reactions have been actively investigated during the last decade in attempts to combine the benefits of click reactions with the excellent spatial and temporal controllability of photochemical processes. ${ }^{[4]}$ UV-induced thiolene and thiol-yne reactions are the most known radical photoclick reactions. ${ }^{[5]}$ Non-radical photoreactions have also attracted a lot of attention in recent years. ${ }^{[6]}$ For instance, Lin et $\mathrm{al}^{[},{ }^{[7]}$ introduced a photo-click 1,3-dipolar tetrazole-ene reaction based on Huisgen's studies. ${ }^{[8]}$ The tetrazole-ene reaction presents several advantages: simplicity of implementation, fast reaction kinetics, high yields, it is catalyst free, yields inoffensive byproducts $\left(\mathrm{N}_{2}\right)$ and, therefore, bio-compatible. This and other photo reactions have been implemented in many different applications such as dendrimers synthesis, ${ }^{[9]}$ bioconjugation, ${ }^{[6 \mathrm{~b}}$, $6 \mathrm{~d}, 6 \mathrm{e}, 10]$ in situ bio-labeling, ${ }^{[6 \mathrm{c}, 11]}$ hydrogels formation, ${ }^{[12]}$ surface functionalization ${ }^{[13]}$ etc. Nevertheless, the implementation of photo reactions in bio-applications is still limited, partly because unnatural functional groups have to be first introduced to a biomolecule. In addition, only a few photo reactions could be applied for polymer-polymer coupling and site-selective

[*] W. Feng, ${ }^{[+]}$Dr. L. Li, ${ }^{[+]}$Dr. P. A. Levkin

Institute of Toxicology and Genetics (ITG)

Karlsruhe Institute of Technology (KIT)

76344 Karlsruhe (Germany)

Fax: (+) 49-721-608-29040

E-mail: levkin@kit.edu

W. Feng, Dr. L. Li, Prof. Dr. O. Trapp

Department of Organic Chemistry

University of Heidelberg,

69120 Heidelberg (Germany)

Chengwu Yang, Dr. A. Welle

Institute of Functional Interfaces (IFG)

KIT (Germany)

Dr. A. Welle

Karlsruhe Nano Micro Facility (KNMF)

$\left.{ }^{+}\right]$These authors contributed equally to this work. conjugation of biomolecules on surfaces. ${ }^{[13 b, 14]}$ Thus, despite a progress in the field of photo-induced reactions, there is a clear need for novel efficient photo reactions that are selective to different types of functionalities, compatible with polymerpolymer conjugation and bioapplications.

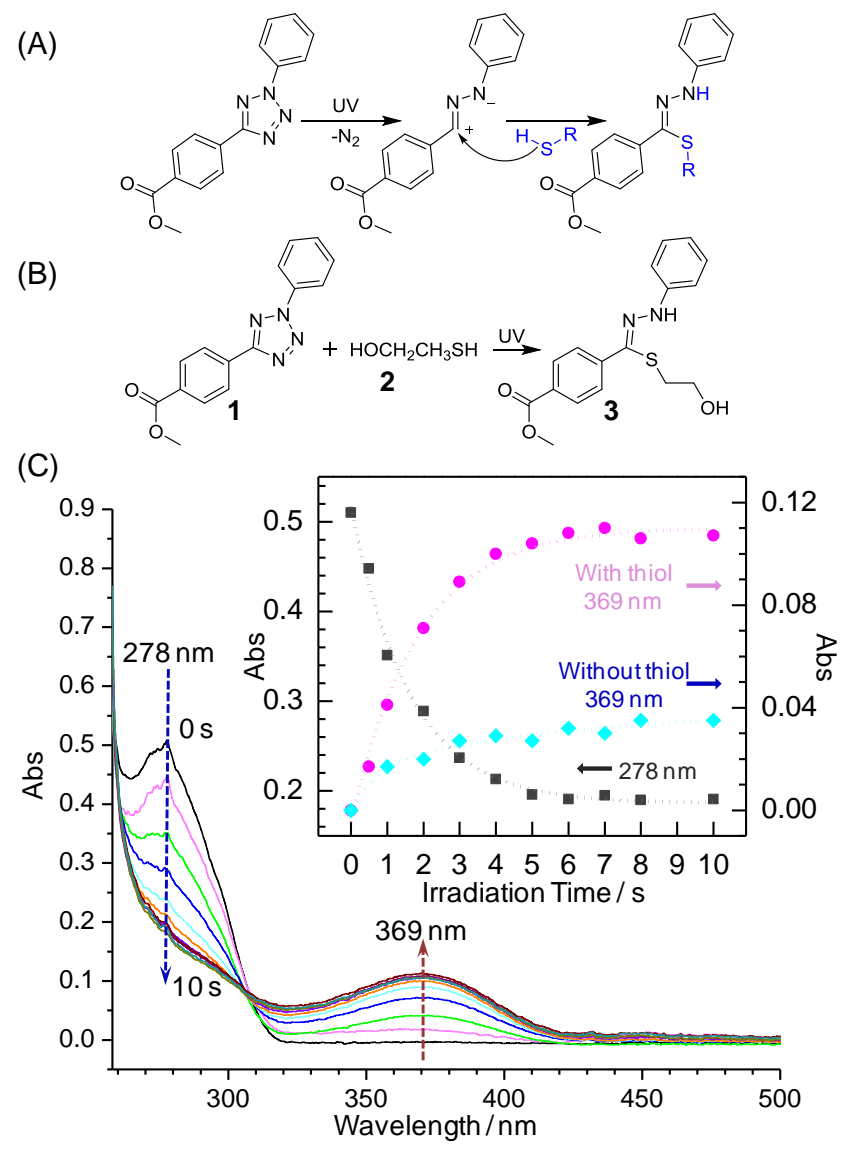

Figure 1. Schematic representation of (A) UV-induced formation of the nitrilimine intermediate from tetrazole $\mathbf{1}$ and subsequent nucleophilic thiol addition; (B) the UV-induced tetrazole-thiol reaction between tetrazole $\mathbf{1}$ and thiol 2. (C) UV-Vis absorbance of the tetrazole-thiol reaction mixture as a function of UV irradiation time. The evolution of the absorbance peaks at 278 $\mathrm{nm}(\bullet)$ as well as the $369 \mathrm{~nm}(\bullet)$ with irradiation time (inset). The control experiments (369 $\mathrm{nm}$ peak is shown as $\downarrow$ ) were taken under the same conditions but without 2 .

50 years ago Huisgen et al. reported that thiophenol could be added to the intermediate nitrilimine generated from decomposition of 2,5-diphenyltetrazole in boiling thiophenol. ${ }^{[8 a, 8 b]}$ Photolytic decomposition of tetrazoles with release of nitrogen and nitrilimines was also described. ${ }^{[8]}$ In this work, we report a UV-induced tetrazole-thiol reaction (Figure $1 \mathrm{~A}$ ) that allows for rapid catalyst-free polymer-polymer conjugation, efficient surface functionalization and patterning as well as opens the way to direct functionalization of biomolecules bearing periphery thiol groups. 
In order to initially examine the kinetics of the UV-induced tetrazole-thiol reaction, a solution of methyl 4-(2-phenyl-2Htetrazol-5-yl)benzoate $\mathbf{1}$ in ethyl acetate and 5 eq. of 2 mercaptoethanol 2 were subjected to irradiation at $260 \mathrm{~nm}$ UV light (Figure 1B). The results showed the UV-triggered rupture of the tetrazole ring evidenced by the gradual decrease in the tetrazole absorption at $278 \mathrm{~nm}$. At the same time a new absorption band around $369 \mathrm{~nm}$ was observed only in the presence of a thiol, confirming the formation of a tetrazole-thiol adduct (Figure 1C-D and S1). Both steps were rapid, achieving complete conversion within $10 \mathrm{~s}$ based on UV-Vis spectroscopy. The resulting tetrazole-thiol adduct, meanwhile, showed a strong fluorescent emission band at $480 \mathrm{~nm}$ (Figure S1). The thiohydrazonate structure of the product 3 was confirmed by ESI-MS and NMR (Figure S2-S4). The kinetics of the UVinduced reaction was also investigated by ${ }^{1} \mathrm{H}$ NMR as shown in Figure S5.

Table S1 shows isolated reaction yields after UV irradiation of tetrazole $\mathbf{1}$ in the presence of thiol $\mathbf{2}$ in both polar protic (ethanol) and aprotic (ethyl acetate) solvents under 260, 312 and $365 \mathrm{~nm}$ UV light. Since the photoactivity of diaryltetrazoles depends on their UV absorption properties ${ }^{[15]}$ and $\mathbf{1}$ absorbs strongly at shorter wavelength region $\left(\lambda_{\max }=278 \mathrm{~nm}\right), 260 \mathrm{~nm}$ and $312 \mathrm{~nm}$ irradiation afforded highest isolated yields in the range of $75 \%$ $95 \%$. The reaction between 1 and equimolar amount of 2 in ethanol leads to $87 \%$ of the isolated product. The thiols could be used in this reaction even in the presence of amine ${ }^{[16]}$, another nucleophile, in polar protic solvents. The tetrazole-thiol adduct 3 was isolated with the yield of $70 \%$ from a mixture of 1 with 2 (2.5 eq.) and ethanolamine (2.5 eq.) after UV irradiation (Figure S6).

Most of surface immobilization or chemical modification of biomolecules require aqueous condition to avoid possible protein denaturation and loss of activity. To demonstrate that UV-induced tetrazole-thiol reaction can proceed in water, a tetrazole-bearing poly(ethylene glycol)methyl ether (MW 5000 $\mathrm{g} / \mathrm{mol}$ ) (PEG-tetrazole 4, Figure S7) was used to react with 2 (5 eq.) in water under $312 \mathrm{~nm}$ UV light for $3 \mathrm{~h}$. The conversion of 4 into the corresponding thiohydrazonate was as high as $95 \%$ based on NMR (Figure S8). In addition, bovine serum albumin (BSA), a protein containing one free peripheral thiol cysteine groups $^{[17]}$, was reacted with $\mathbf{4}$ in aqueous PBS buffer under 312 $\mathrm{nm}$ UV light. The presence of a fluorescent higher molecular weight band in the gel electrophoresis of the product confirmed BSA-PEG conjugation in aqueous medium (Figure S9).

We further investigated the efficiency of the UV-induced tetrazole-thiol reaction for macromolecular conjugation. Figure $2 \mathrm{~A}$ shows a model polymer conjugation experiment that was conducted. O-(2-mercaptoethyl)-O'-methylpolyethylene glycol $\mathbf{5}$ (MW $5000 \mathrm{~g} / \mathrm{mol}$ ) was utilized as the thiol-terminated polymer. Equimolar amounts of $\mathbf{4}$ and $\mathbf{5}$ were dissolved in THF $(3 \mathrm{mg} / \mathrm{mL})$ and subsequently irradiated with $U V_{\lambda=260 \mathrm{~nm}}$ light. The reaction mixture was analyzed by GPC at different irradiation times (Figure 2B). A distinct shift of the GPC traces to lower elution volumes indicates successful formation of a polymer-polymer conjugate 6 already after 7 min of UV irradiation. The remaining small GPC peak corresponding to the starting material could be
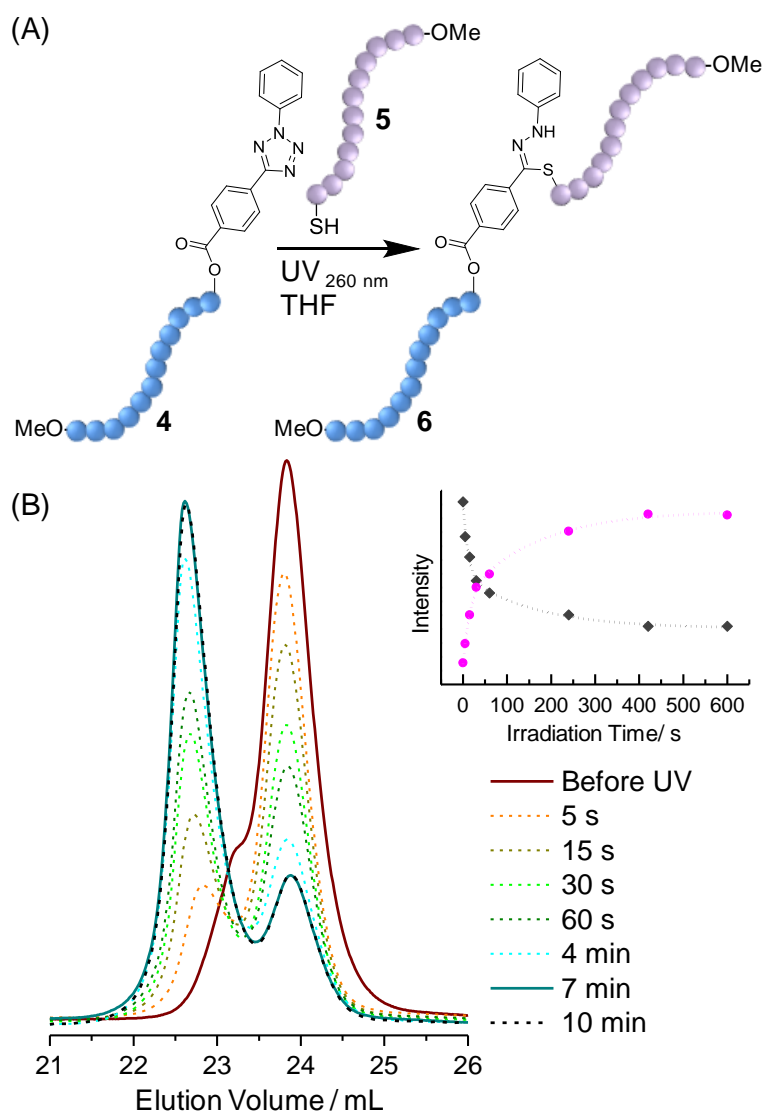

Figure 2. (A) UV-induced conjugation of two polymers using the tetrazole-thiol reaction to form PEG-block-PEG copolymer 6. (B) GPC monitoring of the block-copolymer formation. Evolution of the intensity of elution volumes at 23.8 $\mathrm{mL}(\bullet$, gray) and $22.6 \mathrm{~mL}(\bullet$, pink) with irradiation time (inset).

due to the incomplete tetrazole functionalization of the $\mathbf{4}$ as well as the non-equimolar ratio of reactant.

Next, the performance of the UV-induced tetrazole-thiol reaction for surface functionalization was also examined. A porous polymer layer functionalized with tetrazole (tetrazole surface, Figure S10) was applied as the substrate. With the aid of a photomask, the surface was site-selective modified by $1 \mathrm{H}, 1 \mathrm{H}, 2 \mathrm{H}, 2 \mathrm{H}$-perfluorodecanethiol 7 under $\mathrm{UV}_{\lambda=260 \mathrm{~nm}}$ irradiation (Figure $3 \mathrm{~A}$ ). The successful immobilization and patterning of thiol was confirmed by time-of-flight secondary ion mass spectrometry (ToF-SIMS) (Figure $3 \mathrm{~B}$ and S11). X-ray photoelectron spectroscopy revealed the presence of $F 1 s, F$ $\mathrm{KLL}$ as well as $S 2 p$ peaks after thiol modification. Integration of $\mathrm{N} 1 \mathrm{~s}$ and $\mathrm{S} 2 \mathrm{p}$ peak areas revealed the evolution of conversion with UV irradiation time reaching $88 \%$ after $20 \mathrm{~min}$ of UV irradiation (Figure S12). After tetrazole-thiol reaction, the appearance of fluorescence provides a visualization method to assess the success of surface modification (Figure $3 C$ ). Tetrazole surface modification with a thiol-containing fluorophore, Rhodamine-SH, was also shown in Figure 3D. The produced pattern shows a perfect superimposition of both red and green fluorescence. Surface micropatterns with feature sizes down to $10 \mu \mathrm{m}$ could be obtained using an appropriate photomask (Figure S13). 


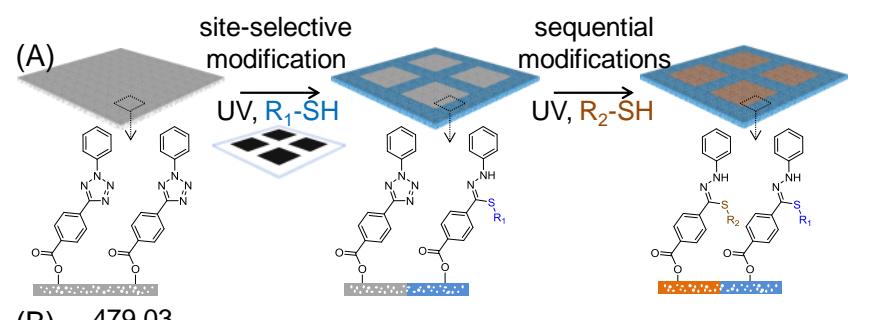

(B) 479.03
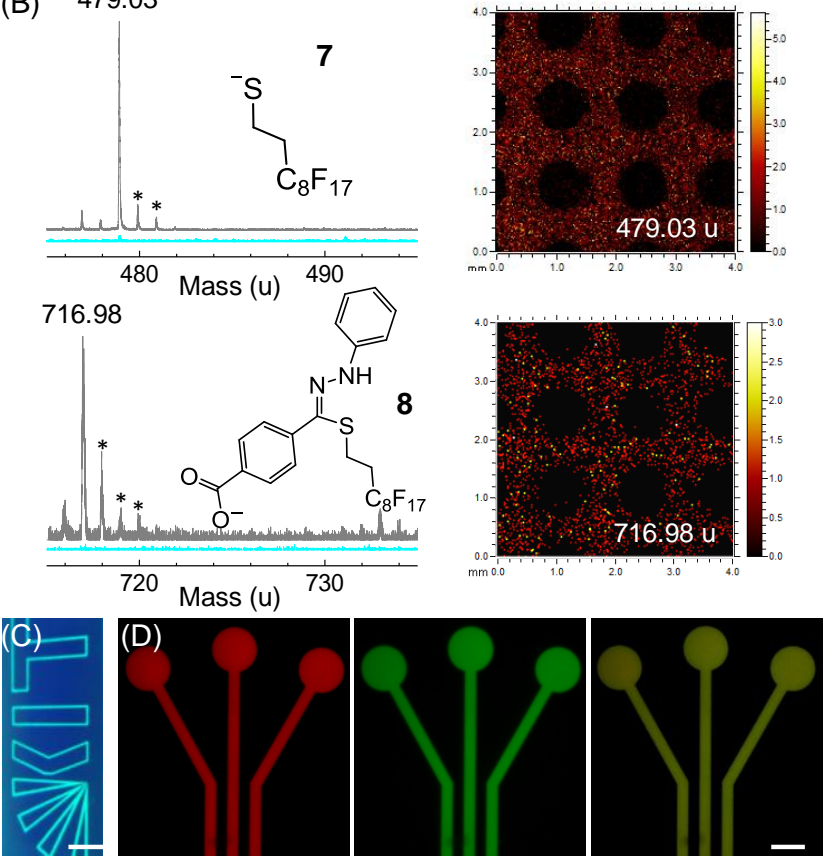

Figure 3. (A) Schematic showing surface micropatterning via the UV-induced tetrazole-thiol reaction. (B) ToF-SIMS (negative polarity) spectra of the tetrazole surface before (blue line) and after (dark line) functionalization with thiol 7. Isotopic peaks are marked with asterisks. The ToF-SIMS images (fragments $479.03 \mathrm{u}$ and $716.98 \mathrm{u}$, corresponding to the thiol 7 ion and the conjugation product $\mathbf{8}$, respectively) of the polymer layer are inserted. Scale bars: $1 \mathrm{~mm}$. (C) Photograph of a thiol 7 patterned tetrazole surface under 365 nm UV light. Scale bar: $3 \mathrm{~mm}$. (D) Red (left), green (middle) and red/green overlay (right) fluorescence microscope images of the tetrazole surface patterned by Rhodamine-SH showing both red and green fluorescence. Scale bar: $300 \mu \mathrm{m}$.

The surface modification could be performed using different thiols in various common solvents (Figure S14). Moreover, when a fluorinated thiol 7 was employed, the hydrophobic tetrazole surface was transformed into a superhydrophobic surface exhibiting water contact angle $\theta_{\text {st }}, \theta_{\text {adv }}$ and $\theta_{\text {rec }}$ as high as $167^{\circ}$, $170^{\circ}$ and $161^{\circ}$, respectively. The SEM analysis in Figure $4 \mathrm{~A}$ did not reveal any changes of the surface morphology after the thiol modification. Hence, the UV-induced tetrazole-thiol reaction could be used to create well-defined superhydrophobichydrophilic micropatterns of different geometries after sequential modifications (Figure 4B and S15). The produced superhydrophobic barriers show good cell repellent properties and mCherry cells only adhered well to the hydrophilic areas (Figure 4C), which is important for a variety of different biotechnological applications ranging from sensors to cell screening microarrays. ${ }^{[18]}$

To demonstrate that the tetrazole-thiol reaction could be used for the in-situ immobilization of biomolecules in aqueous solutions under long-wavelength UV irradiation, we patterned biotin-PEG-thiol onto the tetrazole surface in water under 365 nm UV light (Figure 4D). The surface was then incubated with Alexa Fluor 594 labeled-streptavidin solution. Fluorescence microscopy revealed a two-color green-red fluorescent pattern (Figure 4D), where green fluorescence originated from the thiohydrazonate product of the tetrazole-thiol reaction, while red fluorescence originated from the Alexa Fluor 594 labeledstreptavidin bound to the biotinylated pattern.
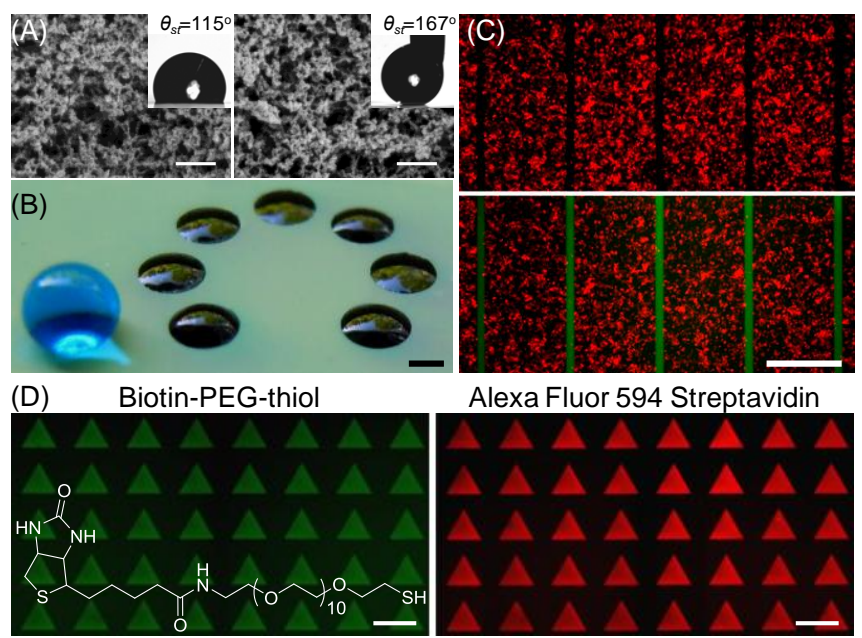

Figure 4. (A) SEM images of the tetrazole surface before (left) and after (right) modification with thiol 7 . The images of a water droplet on the corresponding surfaces are inserted. (B) Optical images of superhydrophobic-hydrophilic micropatterns. (C) Red (top) and red/green overlay (bottom) fluorescence microscope images of the mCherry-expressing rat mammary carcinoma cells after growing for $30 \mathrm{~h}$ on a thiol 7 patterned tetrazole surface. The 7 modified areas emit green fluorescence and show cell repellent properties. (D) Fluorescence microscopy image showing the immobilization of biotin-PEGthiol in water under $365 \mathrm{~nm}$ UV light and Alexa Fluor 594 labeled-streptavidin binding. Scale bars: $500 \mathrm{~nm}(\mathrm{~A})$ and $1 \mathrm{~mm}(\mathrm{~B}-\mathrm{D})$.

In conclusion, we have presented a new versatile UV-induced tetrazole-thiol reaction for conjugation of polymers as well as surface functionalization. The reaction performs very rapidly at ambient temperature with high efficiency and absence of any catalyst. Furthermore, this photo-based approach can be performed in aqueous conditions, making it a promising tool for diverse biological and biotechnological applications, such as protein modification and surface biofunctionalization. The formation of a fluorescent product omits the necessity of using fluorescent labels and can be convenient for tracking the reaction or for multi-color labeling. Because of the above mentioned advantages of this method, we believe that the UVinduced tetrazole-thiol reaction will become a valuable tool for different applications.

\section{Acknowledgements}

This work was funded by the Helmholtz Association's Initiative and Networking Fund (grant VH-NG-621) and European Research Council Starting Grant (ERC-2013-StG 337077DropCellArray). We thank Hualong Yan and Dr. Christine 
Blattner (ITG, KIT) for help for the SDS-PAGE measurements. We are grateful to Prof. Wöll and Dr. Nefedov (IFG, KIT) for their help with XPS measurements.

Keywords: photochemistry - tetrazole-thiol - polymer conjugation $\cdot$ surface modification

[1] a) H. Trommsdorf, Ann. Chem. Pharm. 1834, 11, 190-208; b) H. D. Roth, Angew Chem Int Edit 1989, 28, 1193-1207.

[2] a) H. Okabe, 1978; b) N. J. Turro, Modern molecular photochemistry, University Science Books, 1991; c) S. L. Murov, I. Carmichael, G. L. Hug, Handbook of photochemistry, CRC Press, 1993; d) J. P. Knowles, L. D. Elliott, K. I. Booker-Milburn, Beilstein J. Org. Chem. 2012, 8, 2025-2052; e) K. A. Mosiewicz, L. Kolb, A. J. van der Vlies, M. M. Martino, P. S. Lienemann, J. A. Hubbell, M. Ehrbar, M. P. Lutolf, Nat Mater 2013, 12 , 1072-1078; f) C. Li, A. Glidle, X. Yuan, Z. Hu, E. Pulleine, J. Cooper, W. Yang, H. Yin, Biomacromolecules 2013, 14, 1278-1286; g) S.-H. Lee, J. J. Moon, J. L. West, Biomaterials 2008, 29, 2962-2968; h) T. A. Martin, S. R. Caliari, P. D. Williford, B. A. Harley, R. C. Bailey, Biomaterials 2011, 32 , 3949-3957; i) Y. Li, B. H. San, J. L. Kessler, J. H. Kim, Q. Xu, J. Hanes, S. M. Yu, Macromol Biosci 2015, 15, 52-62.

[3] a) C.-M. Chan, T.-M. Ko, H. Hiraoka, Surface science reports 1996, 24, 154; b) L. Spanhel, M. Haase, H. Weller, A. Henglein, J. Am. Chem. Soc. 1987, 109, 5649-5655; c) Y. Ikada, Biomaterials 1994, 15, 725-736; d) J. A. Rogers, K. E. Paul, R. J. Jackman, G. M. Whitesides, Applied Physics Letters 1997, 70, 2658-2660; e) F. L. Geyer, E. Ueda, U. Liebel, N. Grau, P. A. Levkin, Angew Chem Int Edit 2011, 50, 8424-8427; f) L. Li, J. Li, X. Du, A. Welle, M. Grunze, O. Trapp, P. A. Levkin, Angew Chem Int Edit 2014, 53, 3835-3839.

[4] a) M. A. Tasdelen, Y. Yagci, Angew Chem Int Edit 2013, 52, 5930-5938; b) S. Arumugam, V. V. Popik, J. Am. Chem. Soc. 2012, 134, 8408-8411; c) S. Arumugam, V. V. Popik, J. Am. Chem. Soc. 2011, 133, 5573-5579; d) S. Arumugam, V. V. Popik, J. Am. Chem. Soc. 2011, 133, 15730-15736 e) T. Pauloehrl, G. Delaittre, M. Bruns, M. Meißler, H. G. Börner, M. Bastmeyer, C. Barner-Kowollik, Angew Chem Int Edit 2012, 51, 9181 9184; f) T. Pauloehrl, G. Delaittre, V. Winkler, A. Welle, M. Bruns, H. G. Börner, A. M. Greiner, M. Bastmeyer, C. Barner-Kowollik, Angew Chem Int Edit 2012, 51, 1071-1074; g) T. Pauloehrl, A. Welle, K. K. Oehlenschlaeger, C. Barner-Kowollik, Chem. Sci. 2013, 4, 3503-3507.

[5] a) R. Hoogenboom, Angew Chem Int Edit 2010, 49, 3415-3417; b) A. B. Lowe, C. E. Hoyle, C. N. Bowman, J. Mater. Chem. 2010, 20, 4745-4750; c) C. E. Hoyle, C. N. Bowman, Angew Chem Int Edit 2010, 49, 1540-1573; d) A. Dondoni, Angew Chem Int Edit 2008, 47, 8995-8997.

[6] a) B. J. Adzima, Y. Tao, C. J. Kloxin, C. A. DeForest, K. S. Anseth, C. N. Bowman, Nat Chem 2011, 3, 256-259; b) W. Song, Y. Wang, J. Qu, Q. Lin, J. Am. Chem. Soc. 2008, 130, 9654-9655; c) Z. P. Yu, L. Y. Ho, Q. Lin, J. Am. Chem. Soc. 2011, 133, 11912-11915; d) Y. Wang, W. Song W. J. Hu, Q. Lin, Angew Chem Int Edit 2009, 48, 5330-5333; e) W. Song, Y. Wang, J. Qu, M. M. Madden, Q. Lin, Angew Chem Int Edit 2008, 47, 2832-2835.

[7] R. K. V. Lim, Q. Lin, Acc. Chem. Res. 2011, 44, 828-839.

[8] a) R. Huisgen, R. Grashey, M. Seidel, H. Knupfer, R. Schmidt, Justus Liebigs Annalen der Chemie 1962, 658, 169-180; b) R. Huisgen, J. Sauer, M. Seidel, Chemische Berichte 1961, 94, 2503-2509; c) J. S. Clovis, A. Eckell, R. Huisgen, R. Sustmann, Chemische Berichte 1967, 100, 60-70.

[9] K. L. Killops, L. M. Campos, C. J. Hawker, J. Am. Chem. Soc. 2008, 130 5062-5064

[10] J. Wang, W. Zhang, W. Song, Y. Wang, Z. Yu, J. Li, M. Wu, L. Wang, J. Zang, Q. Lin, J. Am. Chem. Soc. 2010, 132, 14812-14818.

[11] a) Z. Yu, L. Y. Ho, Q. Lin, J. Am. Chem. Soc. 2011, 133, 11912-11915; b) F. Li, H. Zhang, Y. Sun, Y. Pan, J. Zhou, J. Wang, Angew Chem Int Edit 2013, 125, 9882-9886.

[12] a) Y. Fan, C. Deng, R. Cheng, F. Meng, Z. Zhong, Biomacromolecules 2013, 14, 2814-2821; b) C.-C. Lin, A. Raza, H. Shih, Biomaterials 2011, 32, 9685-9695.

[13] a) E. Blasco, M. Piñol, L. Oriol, B. V. K. J. Schmidt, A. Welle, V. Trouillet, M. Bruns, C. Barner-Kowollik, Adv. Funct. Mater. 2013, 23, 4011-4019; b) M. Dietrich, G. Delaittre, J. P. Blinco, A. J. Inglis, M. Bruns, C. BarnerKowollik, Adv. Funct. Mater. 2012, 22, 304-312; c) T. Tischer, C. Rodriguez-Emmenegger, V. Trouillet, A. Welle, V. Schueler, J. O. Mueller, A. S. Goldmann, E. Brynda, C. Barner-Kowollik, Adv. Mater. 2014, 40874092; d) W. Feng, L. Li, E. Ueda, J. Li, S. Heißler, A. Welle, O. Trapp, P. A. Levkin, Adv. Mater. Inter. 2014, DOI: 10.1002/admi.201400269.

[14] a) M. Glassner, K. K. Oehlenschlaeger, T. Gruendling, C. Barner-Kowollik, Macromolecules 2011, 44, 4681-4689; b) K. K. Oehlenschlaeger, J. O. Mueller, N. B. Heine, M. Glassner, N. K. Guimard, G. Delaittre, F. G. Schmidt, C. Barner-Kowollik, Angew Chem Int Edit 2013, 52, 762-766.

[15] a) Y. Wang, W. J. Hu, W. Song, R. K. V. Lim, Q. Lin, Organic Letters 2008 10, 3725-3728; b) Z. Yu, T. Y. Ohulchanskyy, P. An, P. N. Prasad, Q. Lin, J. Am. Chem. Soc. 2013, 135, 16766-16769.

[16] Y. X. Zhang, W. J. Liu, Z. B. K. Zhao, Molecules 2014, 19, 306-315.

[17] a) A. Svenson, J. Carlsson, Biochimica et Biophysica Acta (BBA)-Protein Structure 1975, 400, 433-438; b) M. Oblak, A. Prezelj, S. Pecar, T. Solmajer, Z Naturforsch C 2004, 59, 880-886.

[18] E. Ueda, P. A. Levkin, Adv. Mater. 2013, 25, 1234-1247. 
Supporting Information

\section{UV-induced tetrazole-thiol reaction for polymer conjugation and spatial control of surface functionalization}

Wenqian Feng, Linxian Li, Chengwu Yang, Alexander Welle, Oliver Trapp and Pavel A. Levkin 
Table 1. Isolated yield of the UV-induced 1,3-dipolar nucleophilic addition between $\mathbf{1}$ and $\mathbf{2}$ under different conditions.

\begin{tabular}{cccccc} 
& \multicolumn{2}{c}{$\mathrm{UV}_{260 \mathrm{~nm}}$} & \multicolumn{2}{c}{$\mathrm{UV}_{312 \mathrm{~nm}}$} & $\mathrm{UV}_{365 \mathrm{~nm}}$ \\
$\begin{array}{c}\text { molar ratio } \\
\text { betwen 1 and 2 }\end{array}$ & $1: 1$ & $1: 5$ & $1: 1$ & $1: 5$ & $1: 5$ \\
\hline ethanol & $87 \%$ & $92 \%$ & $87 \%$ & $95 \%$ & $12 \%$ \\
ethyl acetate & $76 \%$ & $88 \%$ & $79 \%$ & $94 \%$ & $28 \%$
\end{tabular}

Table S1 shows isolated reaction yields after UV irradiation of tetrazole $\mathbf{1}$ in the presence of thiol $\mathbf{2}$ in both polar protic (ethanol) and aprotic (ethyl acetate) solvents under 260, 312 and $365 \mathrm{~nm}$ UV light. Since the photoactivity of diaryltetrazoles depends on their UV absorption properties ${ }^{[1]}$ and $\mathbf{1}$ absorbs strongly at shorter wavelength region $\left(\lambda_{\max }=278 \mathrm{~nm}\right), 260 \mathrm{~nm}$ and $312 \mathrm{~nm}$ irradiation afforded highest isolated yields in the range of $75 \%-95 \%$. The reaction between $\mathbf{1}$ and equimolar amount of $\mathbf{2}$ in ethanol leads to $87 \%$ of the isolated product. It is also noteworthy that the thiohydrazonate product could be observed (28\% isolated yield) after irradiation at $365 \mathrm{~nm}$ UV light. In contrast, $365 \mathrm{~nm}$ photoirradiation of $\mathbf{1}$ and methyl methacrylate did not yield any observable pyrazoline cycloadduct by using tetrazole-ene click reaction ${ }^{[1]}$. 
(A)

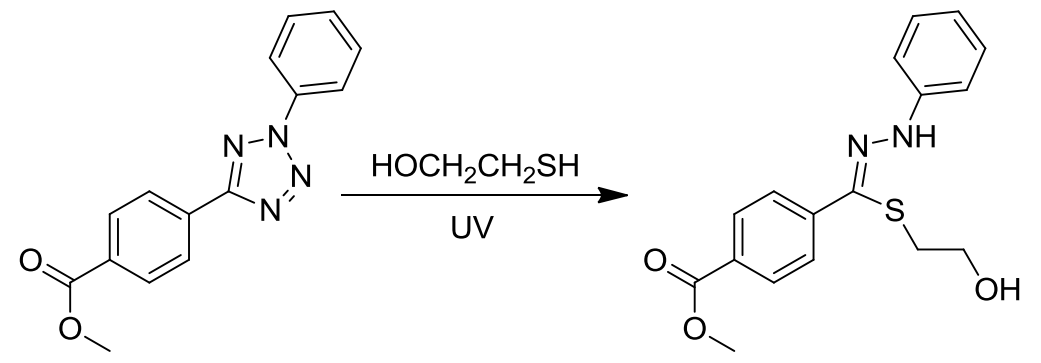

(B)
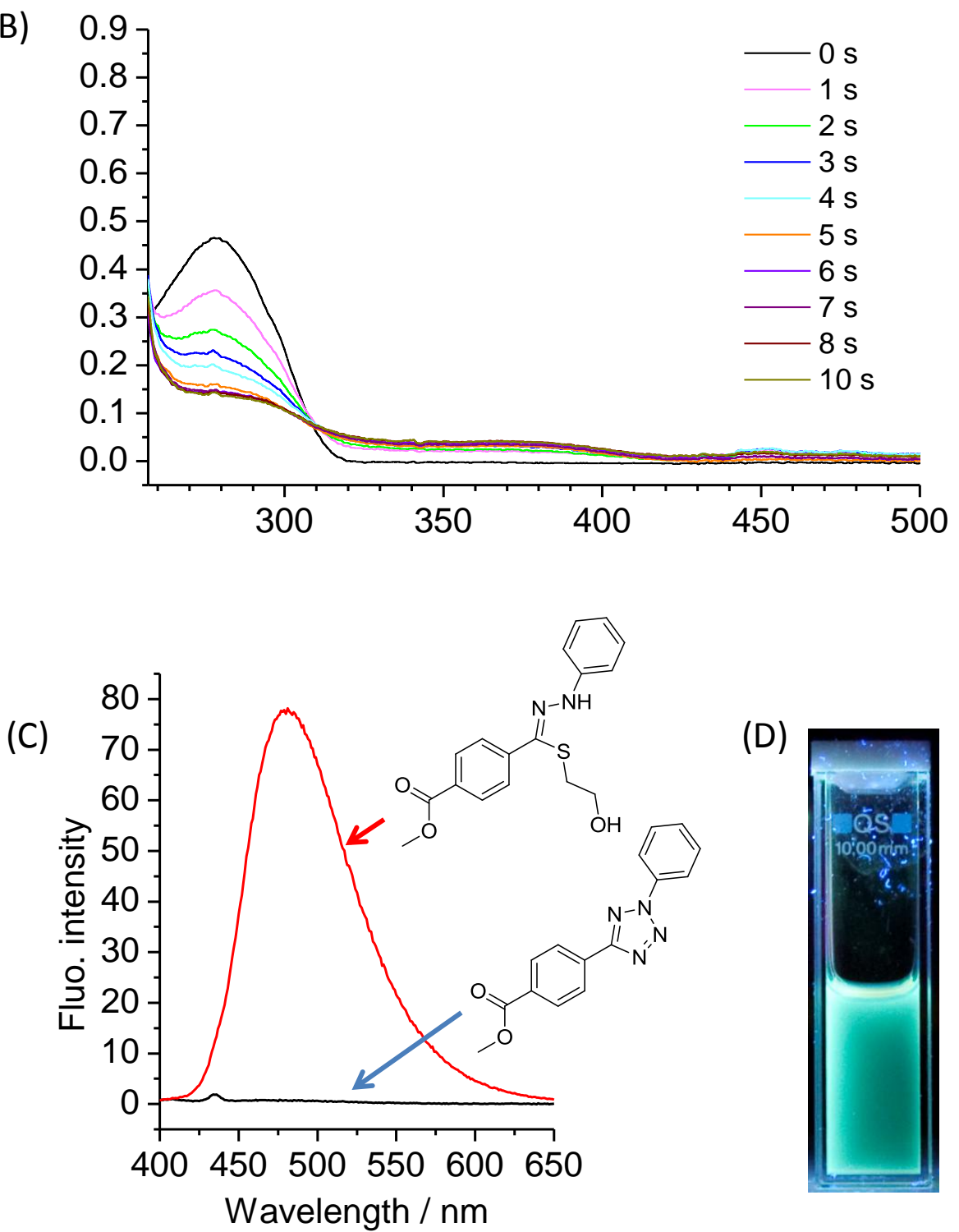

(D)

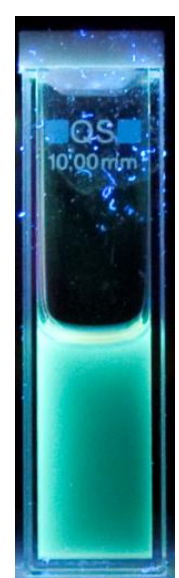


Figure S1. (A) Schematic representation of the UV-induced tetrazole-thiol reaction between methyl 4-(2-phenyl2H-tetrazol-5-yl)benzoate 1 and 2-mercaptoethanol 2. (B) UV-Vis monitoring of the UV-induced docomposition of $20 \mu \mathrm{M} 1$ in ethyl acetate under $260 \mathrm{~nm}$ UV irradiation. (C) The fluorescence spectra of the mixture of $20 \mu \mathrm{M} 1$ and $100 \mu \mathrm{M} 2$ in ethyl acetate before (black line) and after (red line) UV irradiation for $10 \mathrm{~s}$. The photoirradiation wavelength was set at $260 \mathrm{~nm}$ and the excitation wavelength was set at $385 \mathrm{~nm}$. The nucleophilic addition product shows an emission band at $480 \mathrm{~nm}$. (D) Photograph of the thiohydrazonate product $(3 \mathrm{mg} / \mathrm{mL}$ in ethyl acetate) under 365-nm UV light.

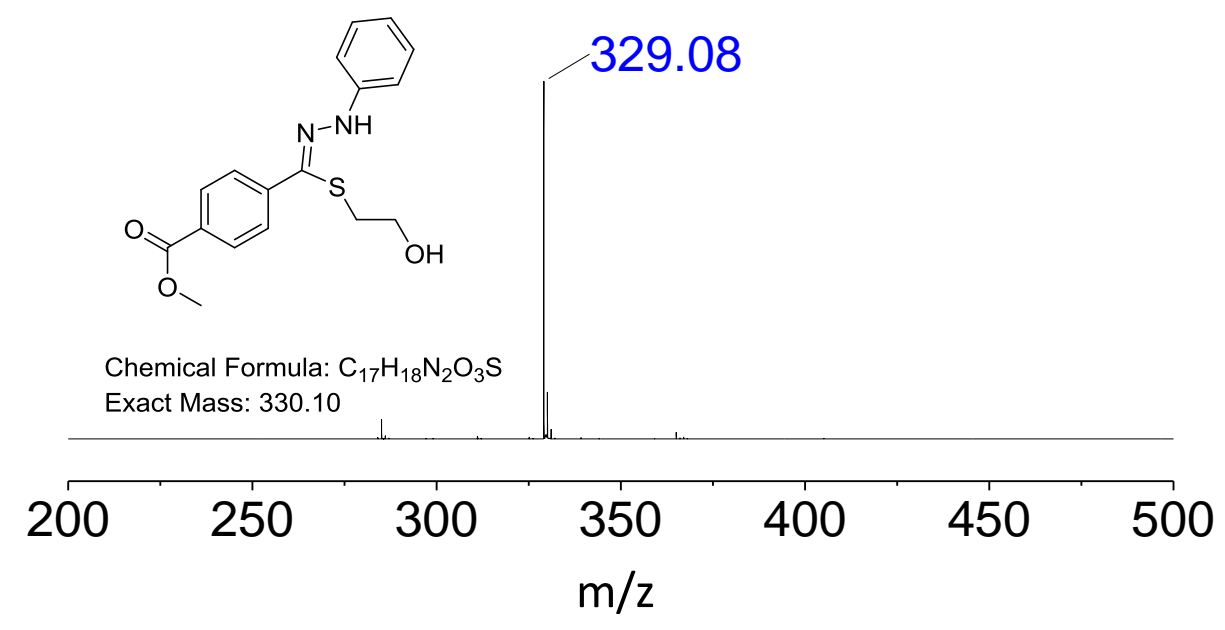

Figure S2. ESI-MS spectrum (negative mode) of the thiohydrazonate product 3, methyl (Z)-4-(((2hydroxyethyl)thio)(2-phenylhydrazono)methyl)benzoate. The nucleophilic tetrazole-thiol addition product, calculated for $\mathrm{C}_{17} \mathrm{H}_{18} \mathrm{~N}_{2} \mathrm{O}_{3} \mathrm{~S}$ 330.10. [M-H]', was found 329.08. 
(A)

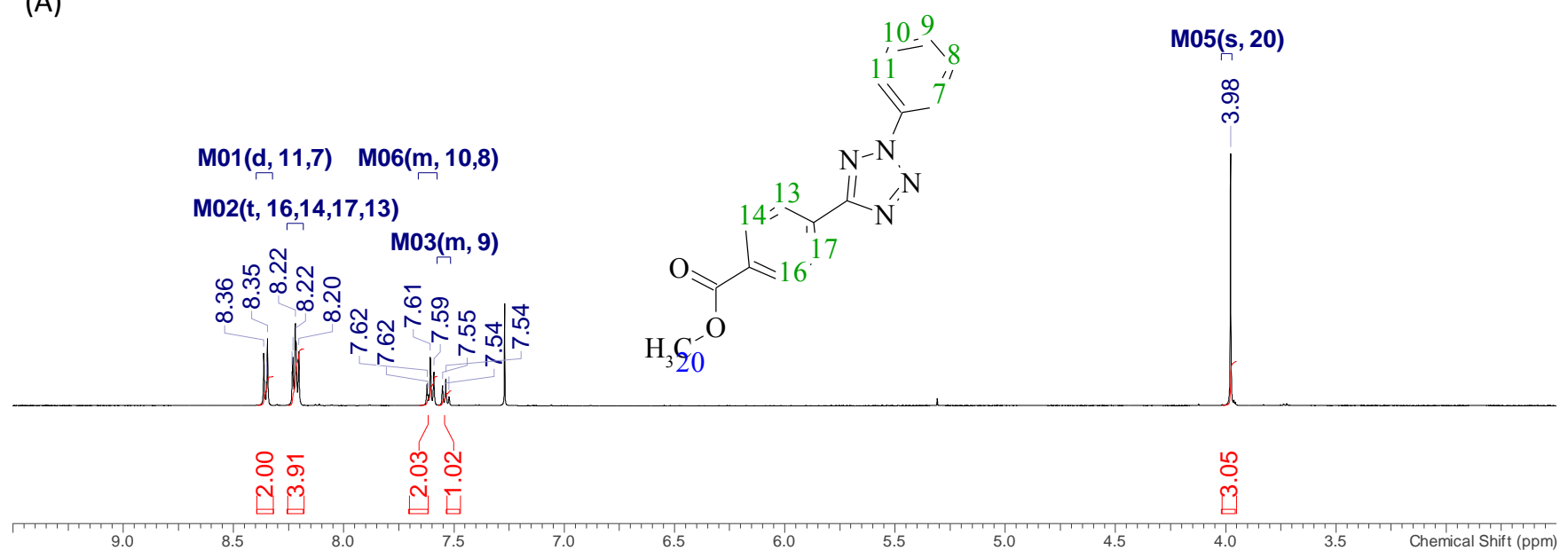

(B)

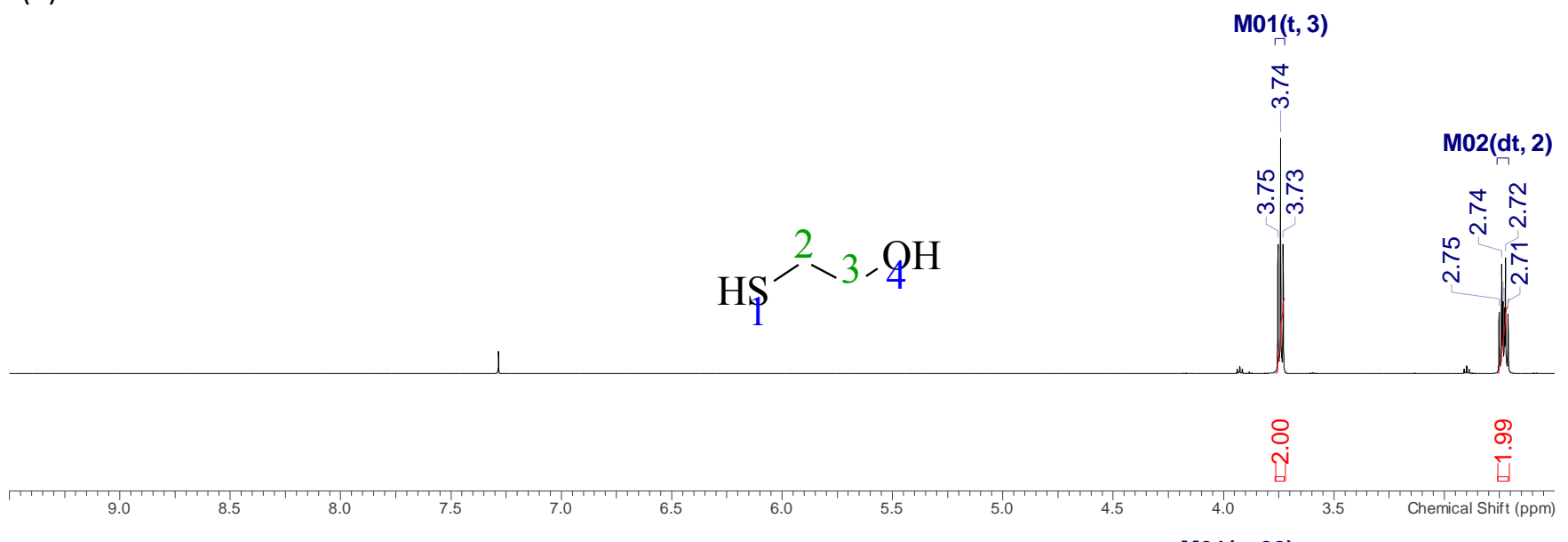

(C)

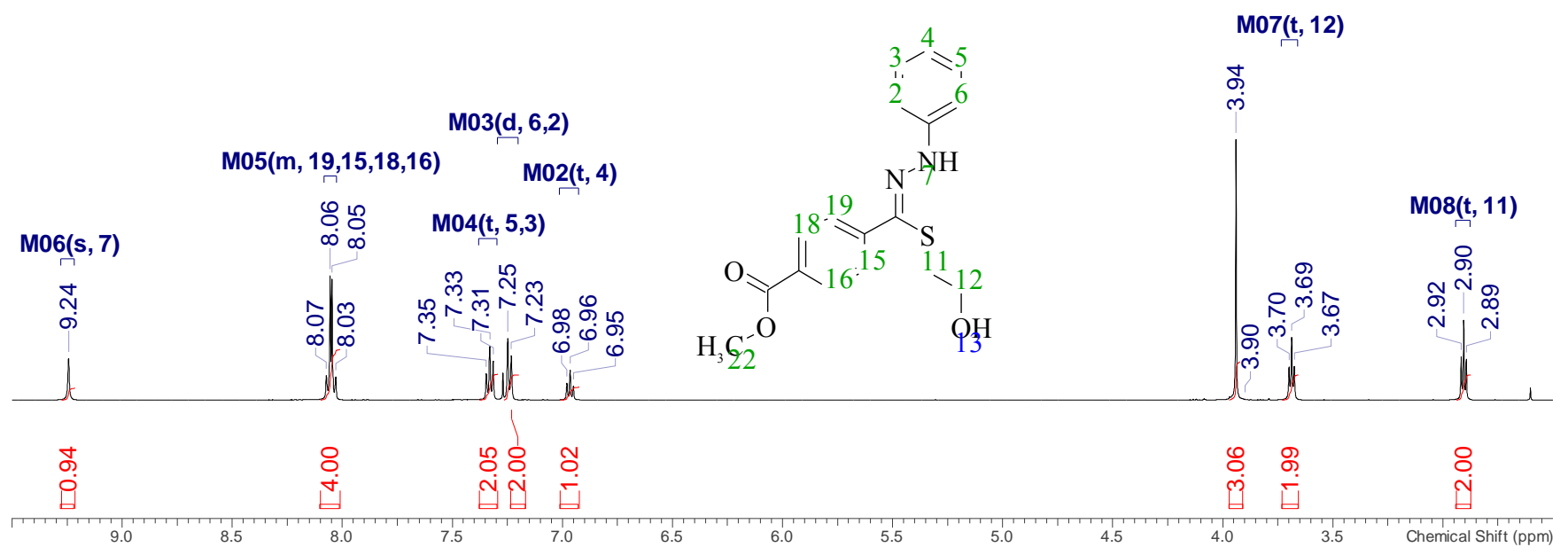




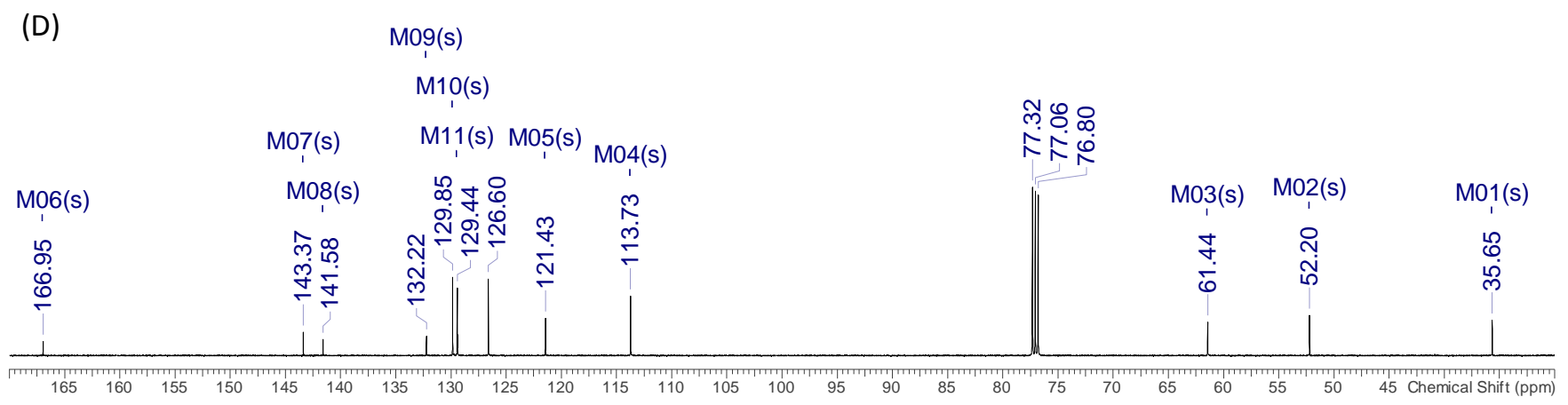

Figure S3. ${ }^{1} \mathrm{H}$ NMR spectra of the reactants (A) methyl 4-(2-phenyl-2H-tetrazol-5-yl)benzoate 1 and (B) 2mercaptoethanol 2, as well as the nucleophilic addition product $3(\mathrm{C})$ formed after UV irradiation. NMR spectra of the product of UV-induced tetrazole-thiol reaction clearly demonstrate the formation of the expected product. First, by comparing the ${ }^{1} \mathrm{H}$ NMR spectrum of 4-(2-phenyl-2H-tetrazol-5-yl)benzoate 1 with the 1,3-dipolar nucleophilic addition product $\mathbf{3}$, there is a clear change in the signals between $\delta=6.5$ and 8.5 ppm that are associated with the aromatic protons. The integration value for the signals arising from the aromatic protons is in perfect agreement with the number of aromatic protons expected for the product, indicating the UV-triggered rupture of the tetrazole. Moreover, the NMR signals associated with the 2-mercaptoethanol's protons shifted to lower field (from $\delta=2.68$ to $\delta=2.86 \mathrm{ppm}$ ). In addition, the sextet corresponding to the $\mathrm{HS}-\underline{\mathrm{C}}_{2}$ - protons changes to a triplet of $-\mathrm{S}-\mathrm{CH}_{2}$, indicating that the 2-mercaptoethanol $\mathbf{2}$ is linked now to the hydrazone. Finally, the presence of a new resonance signal at $\delta=9.24 \mathrm{ppm}$ further suggests that the thiol group reacted with the nitrilimine. ${ }^{[2]}$ (D) ${ }^{13} \mathrm{C}$ NMR spectrum of the thiohydrazonate product 3 . 
(A)

$\underset{n}{\operatorname{M04}(\mathrm{s})}$

Solvent:

EtOAc
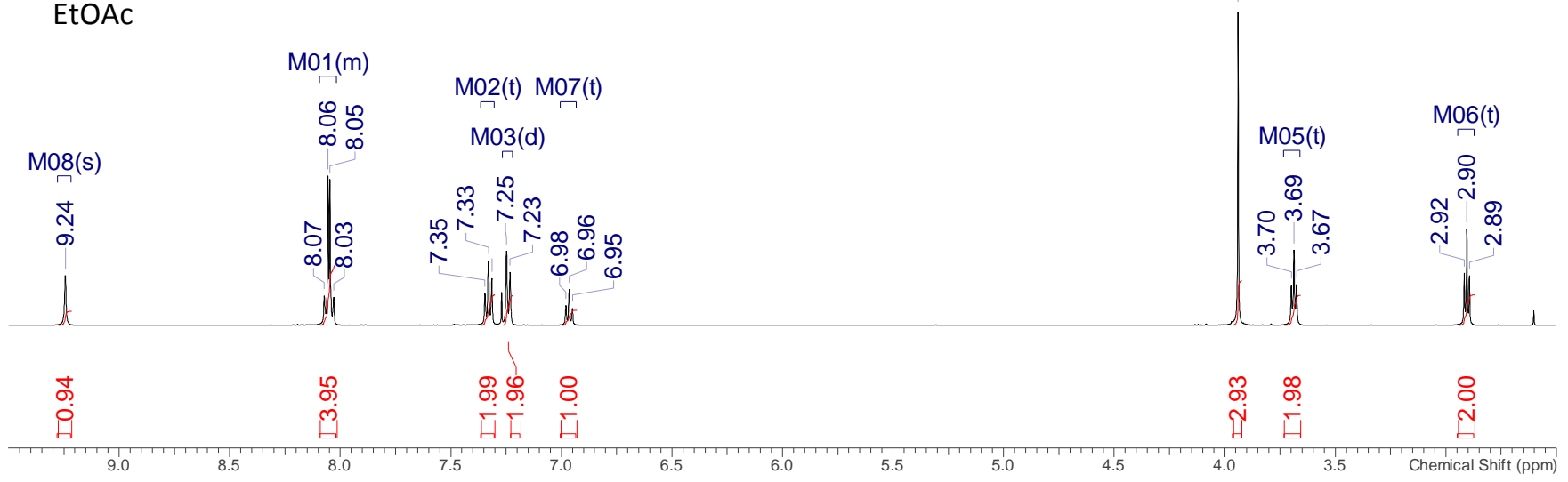

(B)

Solvent:

$\mathrm{EtOH}$

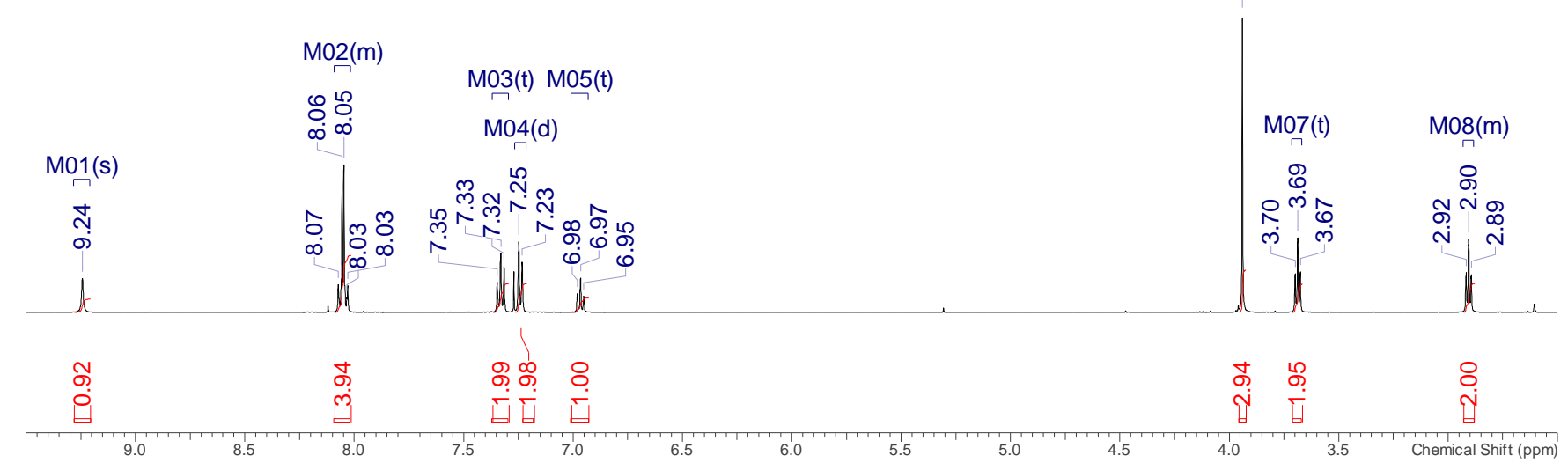

Figure S4. ${ }^{1} \mathrm{H}$ NMR spectra of the thiohydrazonate product $\mathbf{3}$ formed after UV irradiation in ethyl acetate (A) and ethanol (B), respectively. 
(A)

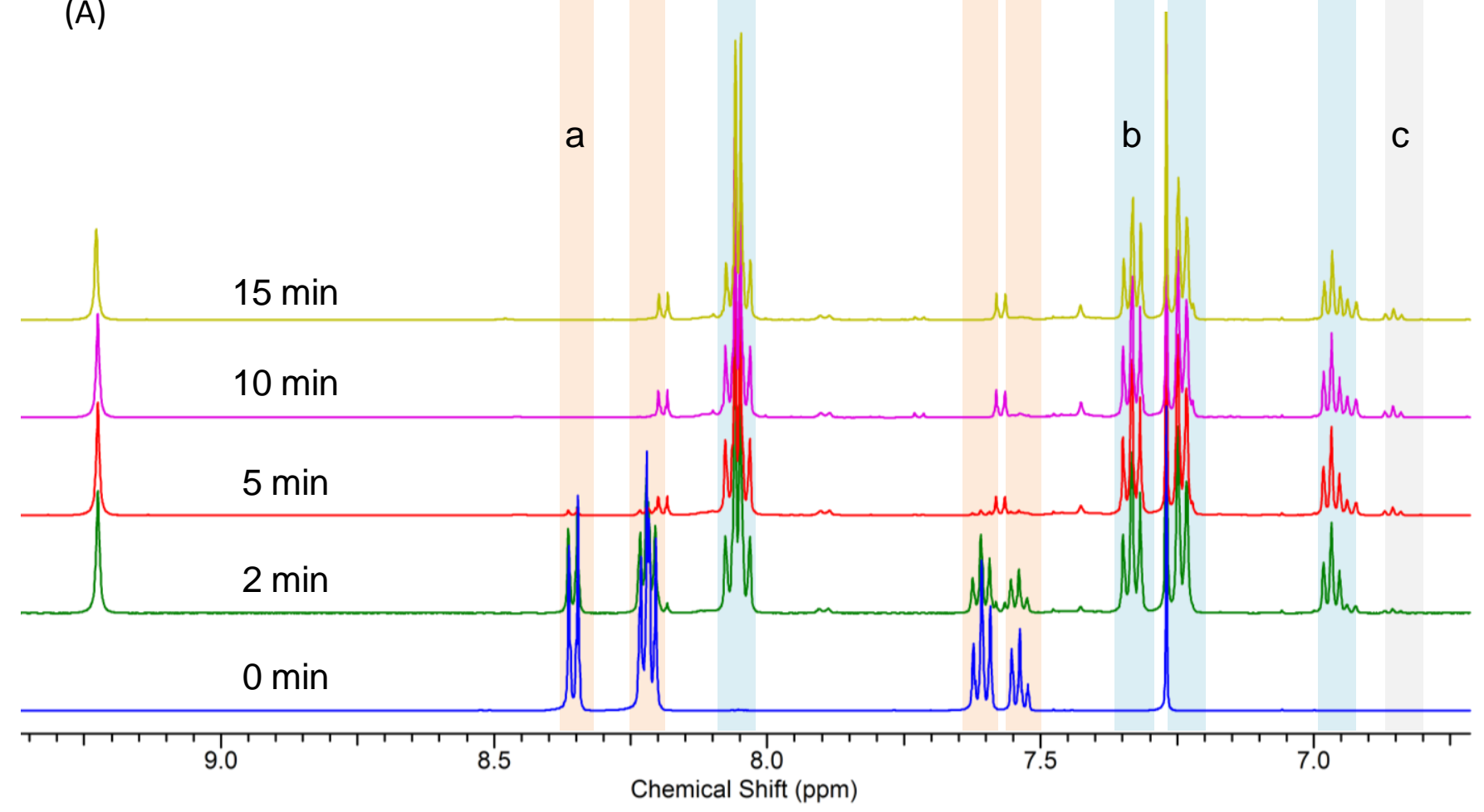

(B)

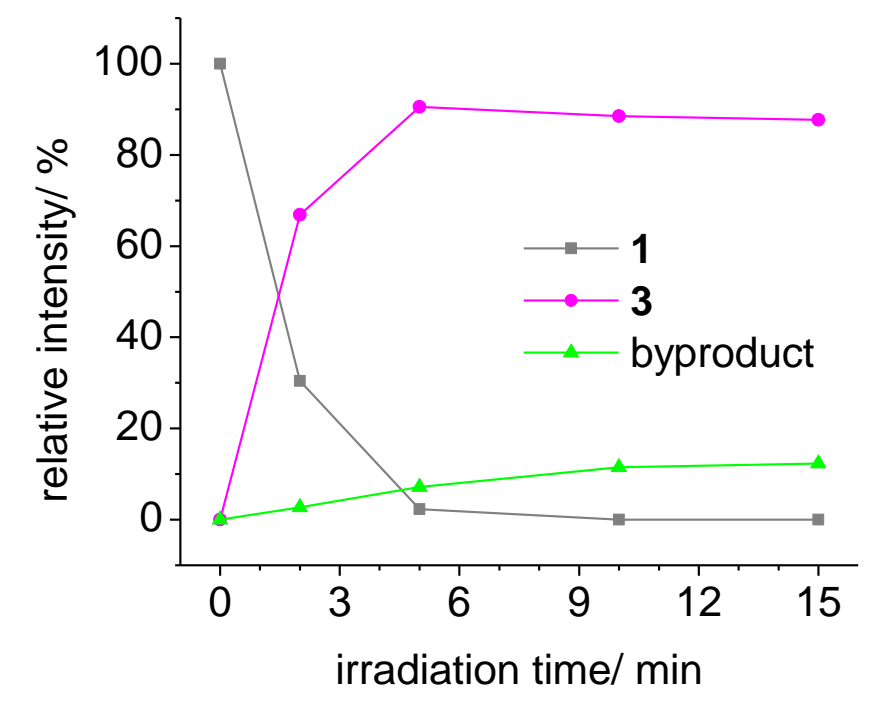

Figure S5. (A) ${ }^{1} \mathrm{H}$ NMR spectra monitoring of reaction progress between methyl 4-(2-phenyl-2H-tetrazol-5yl)benzoate $1(0.75 \mathrm{mg} / \mathrm{mL})$ and 2-mercaptoethanol 2 (5 eq.) in ethyl acetate under $260 \mathrm{~nm}$ UV light (intensity 5 $\mathrm{mW} / \mathrm{cm}^{2}$ ) at intervals of 0, 2, 5, 10,15 min. (B) Evolution of the peak intensity of three major species during the UV irradiation. The NMR spectra clearly demonstrated fast decomposition of reactant $\mathbf{1}$ and formation of 
expected product 3. This result is in agreement with the UV-Vis spectra shown in Figure 1. Relative intensity of each species was obtained from the integration of signals $\mathrm{a}, \mathrm{b}$ and $\mathrm{c}$ associated with $\mathbf{1}, \mathbf{3}$ and a byproduct, respectively. The reactant $\mathbf{1}$ decomposed completely after 5 min irradiation and the yield of thiohydrazonate product 3 reached $91 \%$.

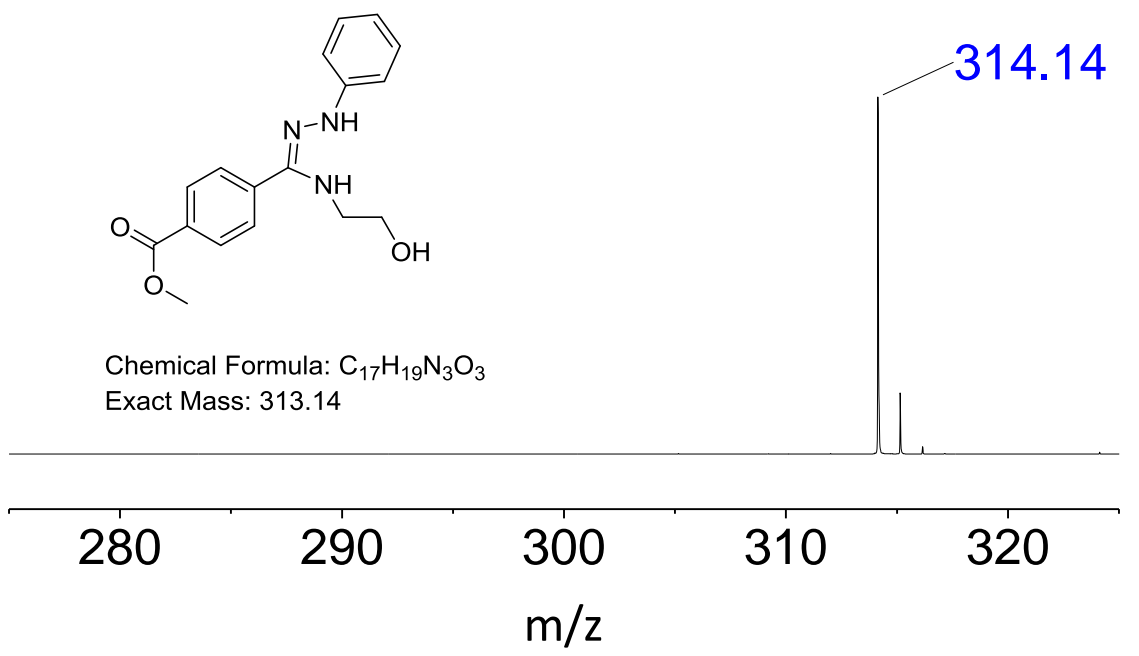

Figure S6. ESI-MS spectra of the nucleophilic addition product between tetrazole 1 and ethanolamine, methyl (Z)-4-(N-(2-hydroxyethyl)-N'-phenylcarbamohydrazonoyl)benzoate. The nucleophilic tetrazole-amine addition product, calculated for $\mathrm{C}_{17} \mathrm{H}_{19} \mathrm{~N}_{3} \mathrm{O}_{3}$ 313.14. $[\mathrm{M}+\mathrm{H}]^{+}$, was found 314.14.

In order to test whether UV-induced tetrazole-thiol reaction can be performed in the presence of other nucleophiles, such as amines, a mixture of 1 with 2 (2.5 eq.) and ethanolamine (2.5 eq.) in ethanol solution was irradiated with $\mathrm{UV}_{\lambda=312 \mathrm{~nm}}$ for $2 \mathrm{~h}$. Although the tetrazole-amine adduct was also detected by MS (Figure S5), the tetrazole-thiol adduct 3 was isolated with the yield of 70\%, showing that thiols can be used in this reaction even in the presence of amines in polar protic solvents. 
(A)

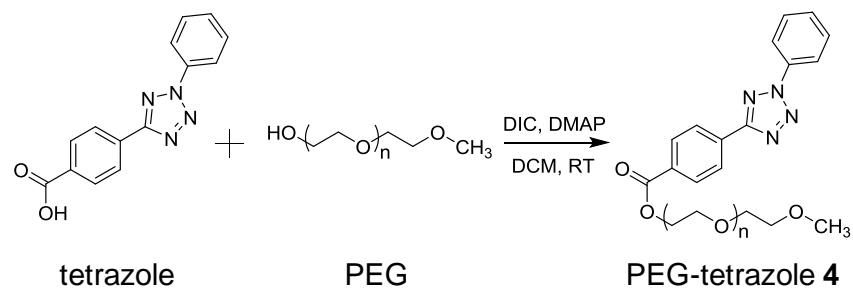

(B)

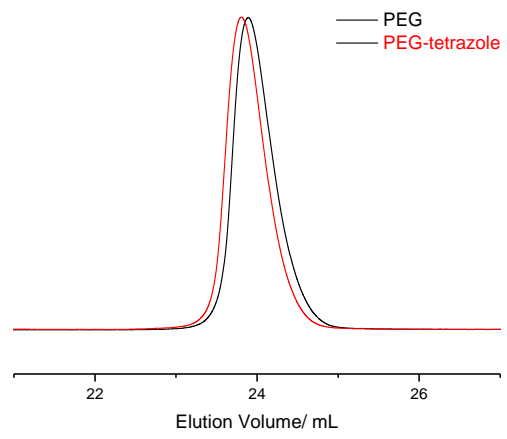

(C)

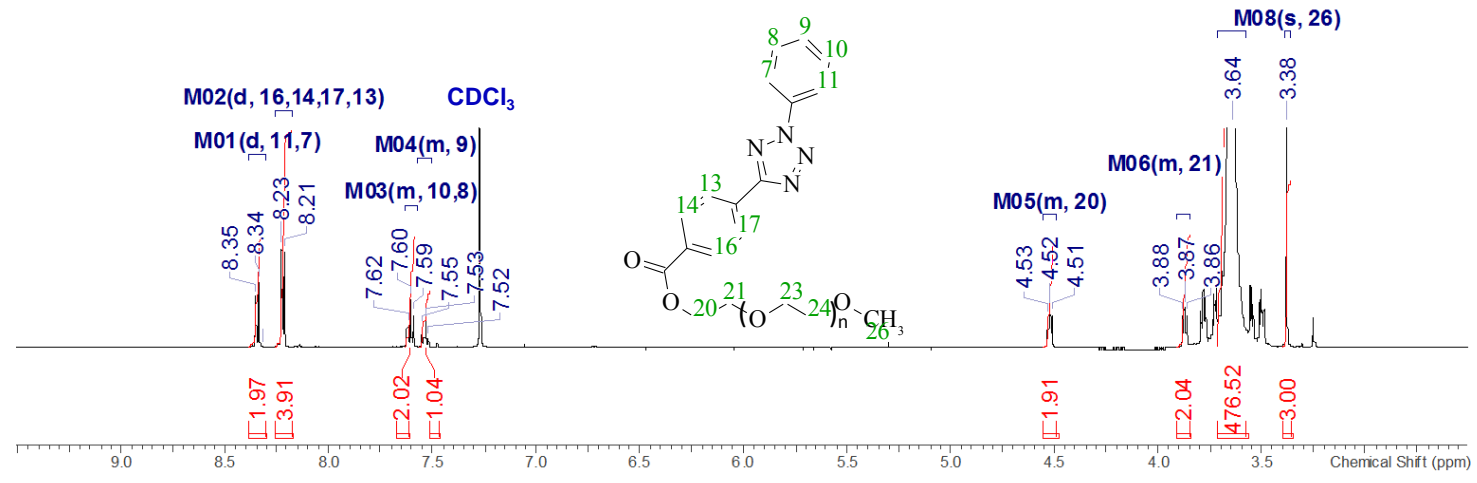

Figure S7. (A) Schematic representation of the formation of PEG-tetrazole 4 via esterification. (B) Overlay of GPC traces (THF) showing the formation of PEG-tetrazole 4 from PEG. The result shows that the PEG-tetrazole 4 polymer peak shifted towards lower elution volumes after the modification indicating an increased molecular weight. (C) ${ }^{1} \mathrm{H}$ NMR spectra of the PEG-tetrazole 4. The presence of the resonance at 4.49 ppm (f), attributed to the PEG-tetrazole methylene protons, confirms the formation of the product. The grafting of PEG with tetrazole is calculated to be about $95 \%$ by comparing the integrations of peaks from the methylene protons of PEG-tetrazole 4 at $4.49 \mathrm{ppm}$ (f) and the methyl protons of the PEG at $3.35 \mathrm{ppm}(\mathrm{i})$. Here the $\mathrm{n} \approx 110$. 
(A)

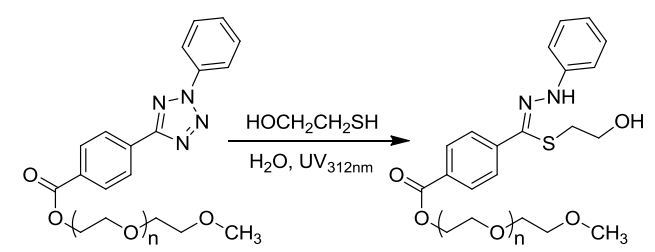

(B)

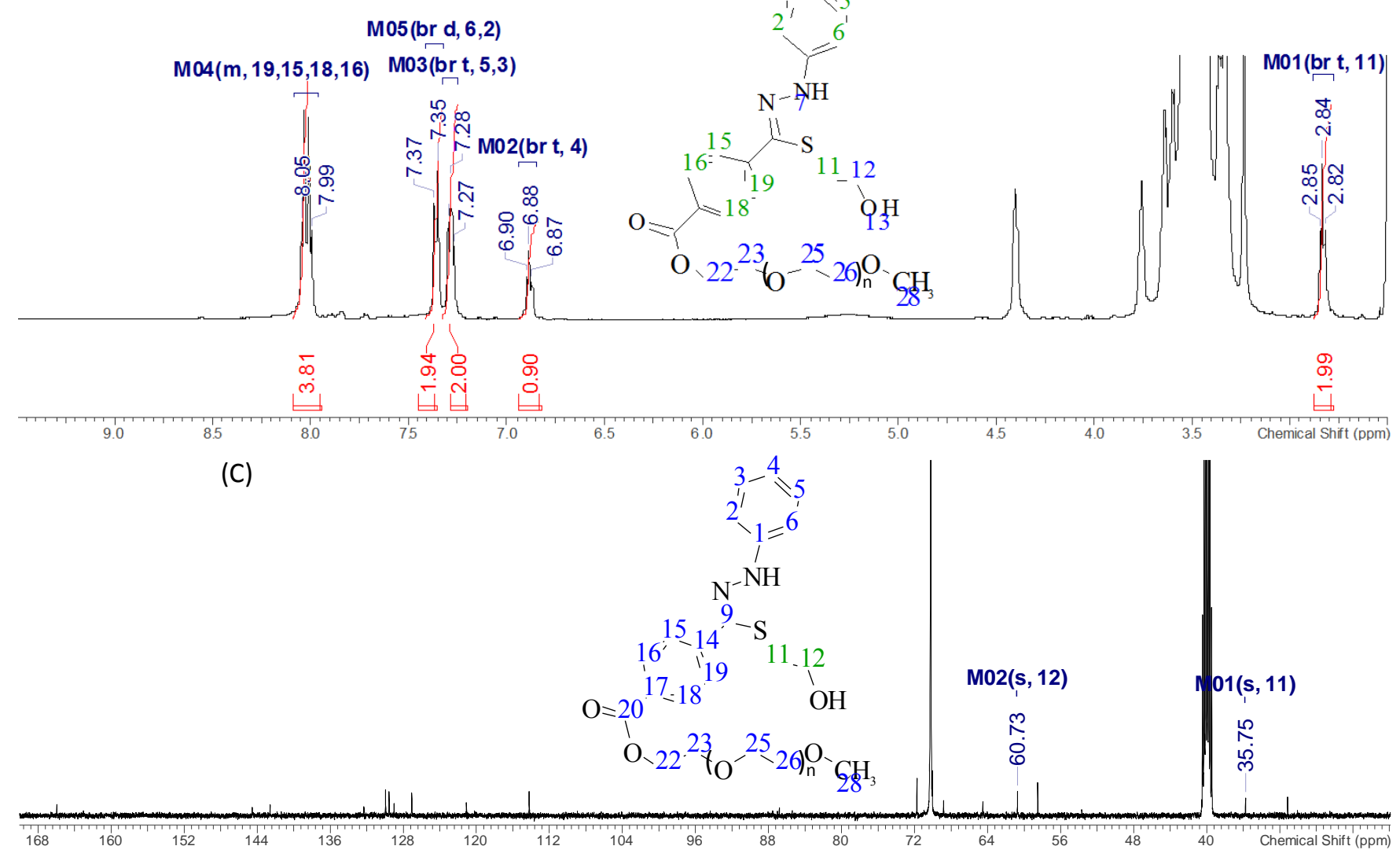

Figure S8. (A) Schematic representation of the UV-induced tetrazole-thiol reaction between PEG-tetrazole 4 and 2-mercaptoethanol 2 in water. (B) ${ }^{1} \mathrm{H}$ NMR spectra of the nucleophilic addition product formed after UV irradiation and purification. The presence of the resonance at $2.84 \mathrm{ppm}$, attributed to the methylene protons from 2-mercaptoethanol, confirms the formation of the nucleophilic addition product in water. The conversion is calculated to be about $95 \%$ by comparing the integrations of peaks from the methylene protons of nucleophilic addition product at $2.84 \mathrm{ppm}$ and the aromatic protons. (C) ${ }^{13} \mathrm{C}$ NMR spectra of the nucleophilic addition product formed after UV irradiation and purification. The presence of the resonances at $35.75 \mathrm{ppm}$ and $60.73 \mathrm{ppm}$, attributed to the methylene protons from 2-mercaptoethanol, confirms the formation of the nucleophilic addition product in water. Here the $n \approx 110$. 
(A)
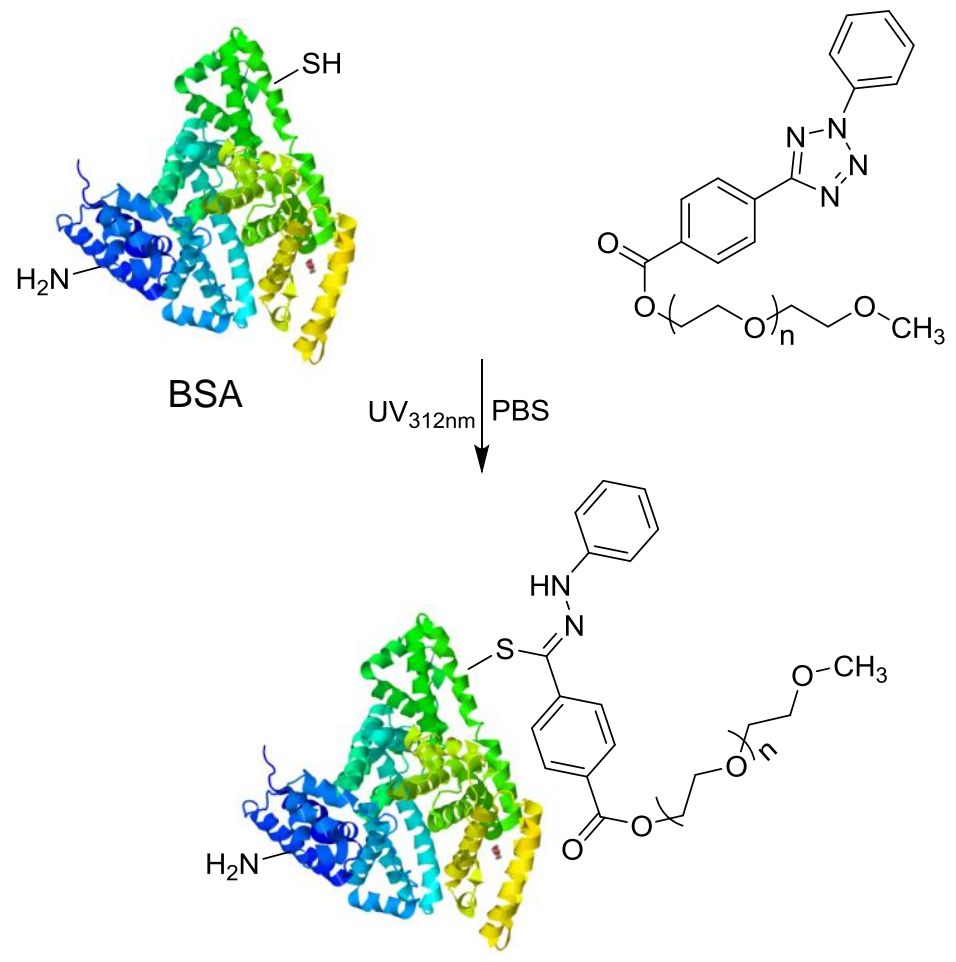

(B)

PEG modified BSA

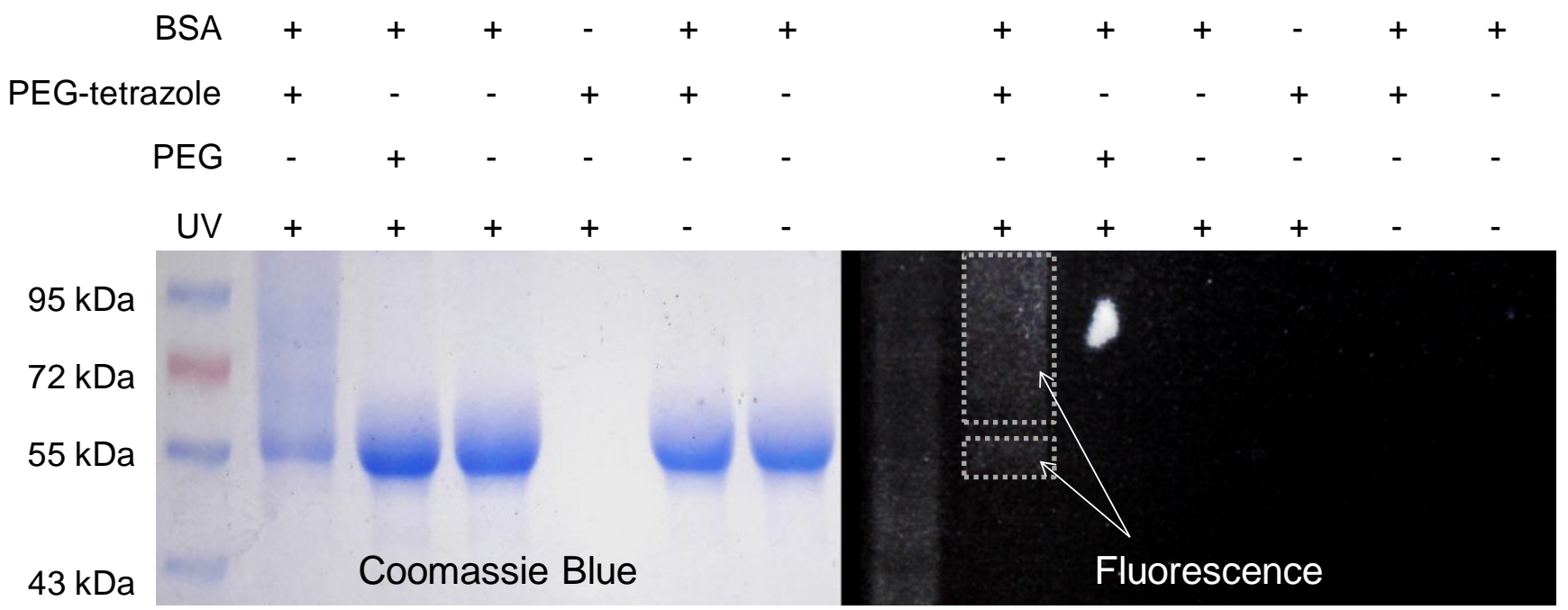

Figure S9. (A) Schematic representation of the bovine serum albumin (BSA) modification by PEG-tetrazole 4 using UV-induced tetrazole-thiol reaction in water. (B) Coomassie Blue staining (left) and in-gel fluorescence imaging (right, $\mathrm{UV}_{\lambda \mathrm{ex}}=365 \mathrm{~nm}$ ) of various samples. Photoinduction was carried out for a duration of 5 min with UV irradiation at $312 \mathrm{~nm}$ and additional incubation for $2 \mathrm{~h}$ in PBS buffer. The experiment was repeated twice with 
similar results. A higher-molecular-weight band was observed in the presence of PEG-tetrazole 4 and UV irradiation after Coomassie Blue staining, suggesting the successful modification of protein by PEG-tetrazole 4 after UV irradiation in the aqueous medium. Meanwhile, the presence of fluorescence further corroborates the successful protein functionalization. Due to the excess PEG-tetrazole $\mathbf{4}$ being used and the existence of amine groups in BSA, part of the protein could be multi-modified by the PEG-tetrazole 4.

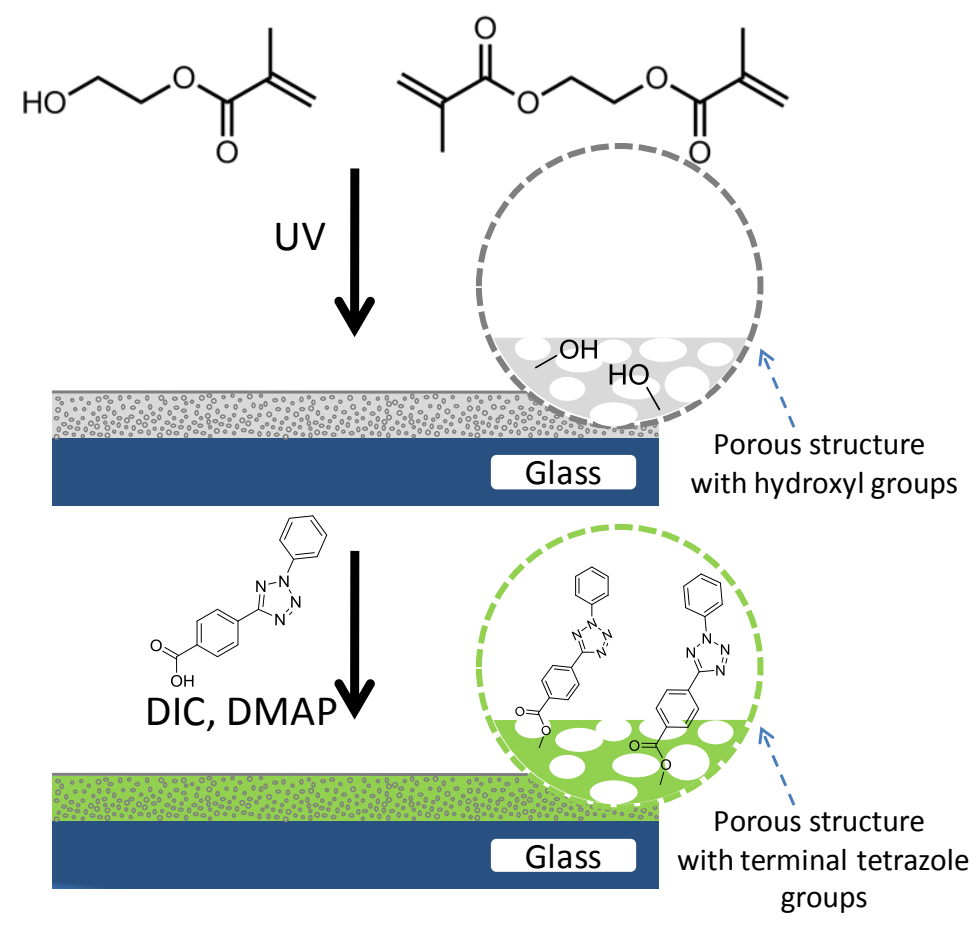

Figure S10. Schematic description of the fabrication of the tetrazole-modified porous polymer surface (tetrazole surface). Firstly, a $12.5 \mu \mathrm{m}$ thin, hydrophilic porous polymer film, poly(2-hydroxyethyl methacrylate-co-ethylene dimethacrylate) (HEMA-EDMA), was prepared on a glass substrate using photo-initiated copolymerization of 2hydroxyethyl methacrylate and ethylene dimethacrylate in the presence of porogens according to the known procedure. ${ }^{[3]}$ The hydroxyl groups were then esterified using 4-(2-phenyl-2H-tetrazol-5-yl)benzoic acid by incubating the surface in a dichloromethane solution of 4-(2-phenyl-2H-tetrazol-5-yl)benzoic acid, coupling reagent $N, N^{\prime}$-diisopropylcarbodiimide (DIC) and catalyst 4-(dimethylamino)pyridine (DMAP) under stirring at room temperature for 24 hours. The hydrophilic HEMA-EDMA surface (static water contact angle $\theta_{\text {st }} 5^{\circ}$ ) was transformed into a hydrophobic tetrazole surface with the $\theta_{\mathrm{st}}$ of $115^{\mathrm{o}} \cdot{ }^{[4]}$ 
(A)

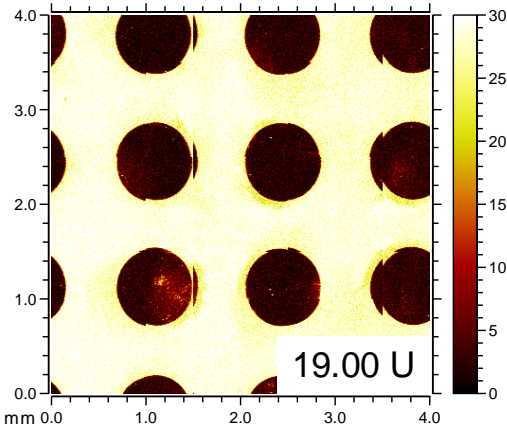

$\mathrm{F}^{-}$

Exact Mass: 19.00

(C)
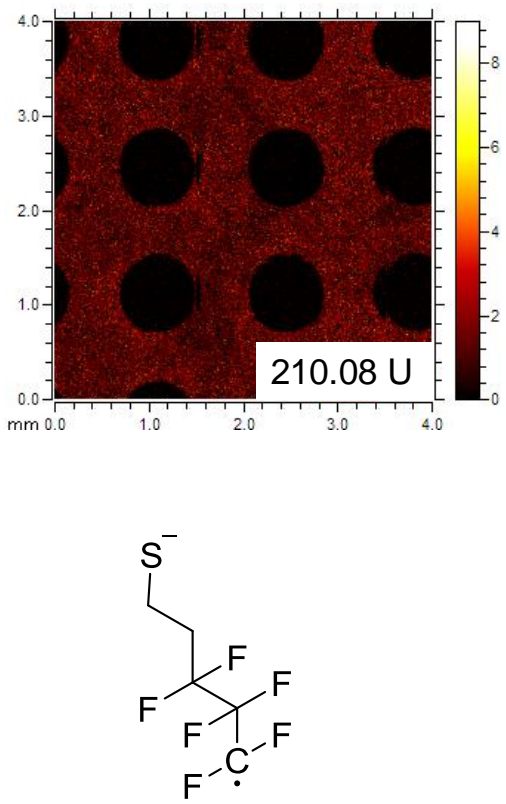

Chemical Formula: $\mathrm{C}_{5} \mathrm{H}_{4} \mathrm{~F}_{6} \mathrm{~S}^{--}$

Exact Mass: $209.99 \mathrm{~g} / \mathrm{mol}$

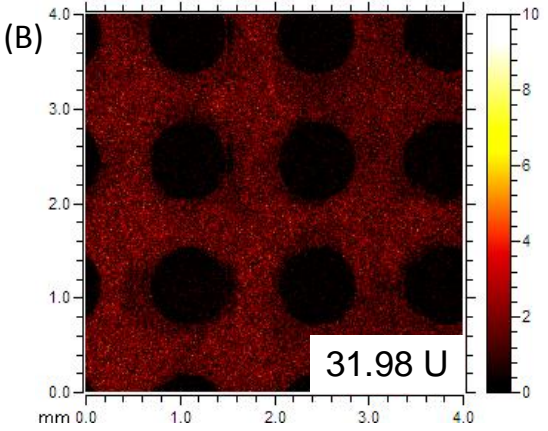

$\mathrm{S}^{-}$

Exact Mass: 31.97

(D)
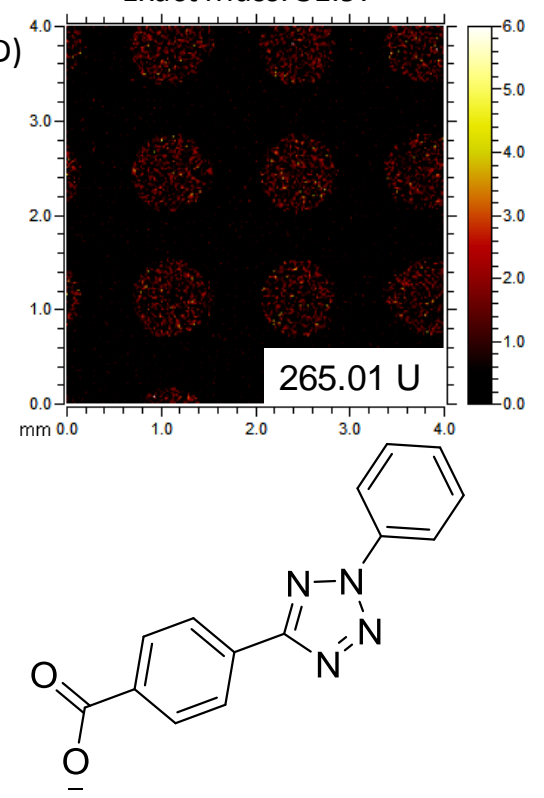

Chemical Formula: $\mathrm{C}_{14} \mathrm{H}_{9} \mathrm{~N}_{4} \mathrm{O}_{2}^{-}$ Exact Mass: $265.07 \mathrm{~g} / \mathrm{mol}$

Figure S11. The ToF-SIMS images of the tetrazole surface after site-selective functionalization with $1 H, 1 H, 2 H, 2 H$-perfluorodecanethiol 7. (A) fragments $19.00 \mathrm{~m} / \mathrm{z}$, corresponding to $\mathrm{F}$; (B) fragments $31.98 \mathrm{~m} / \mathrm{z}$, corresponding to $\mathrm{S}^{-}$; (C) fragments $210.08 \mathrm{~m} / \mathrm{z}$, corresponding to the $\mathrm{C}_{5} \mathrm{H}_{4} \mathrm{~F}_{6} \mathrm{~S}^{-}$, part of $1 H, 1 H, 2 H, 2 H$ perfluorodecanethiol; and (D) fragments $265.01 \mathrm{u}$, corresponding to the 4-(2-phenyl-2H-tetrazol-5-yl)benzoic acid ion.

The tetrazole surface was wetted with an ethyl acetate solution containing $20 \mathrm{vol} \%$ of $1 \mathrm{H}, 1 \mathrm{H}, 2 \mathrm{H}, 2 \mathrm{H}$ perfluorodecanethiol 7 and irradiated with $\mathrm{UV}_{\lambda=260 \mathrm{~nm}}$ through a quartz photomask for 2 min (site-selective 
modification, Figure 3A). The successful immobilization of thiol was confirmed by time-of-flight secondary ion mass spectrometry (ToF-SIMS) (Figure 3B and S10). Both of the peaks corresponding to thiol ion $7(\mathrm{~m} / \mathrm{z}=479.03)$ as well as the corresponding conjugation product $\mathbf{8}$ at 716.98 were detected in the negative polarity mode (Figure 3B). The lateral distributions of these two signals are shown in Figure 3B. Figure S10 also shows clear patterns with good contrast of the $\mathrm{F}^{-}, \mathrm{S}^{-}$and $\mathrm{C}_{5} \mathrm{H}_{4} \mathrm{~F}_{6} \mathrm{~S}^{-}$ions. The tetrazole ion only appeared on the non-irradiated areas, indicating the photolysis of tetrazole on the exposed area. 

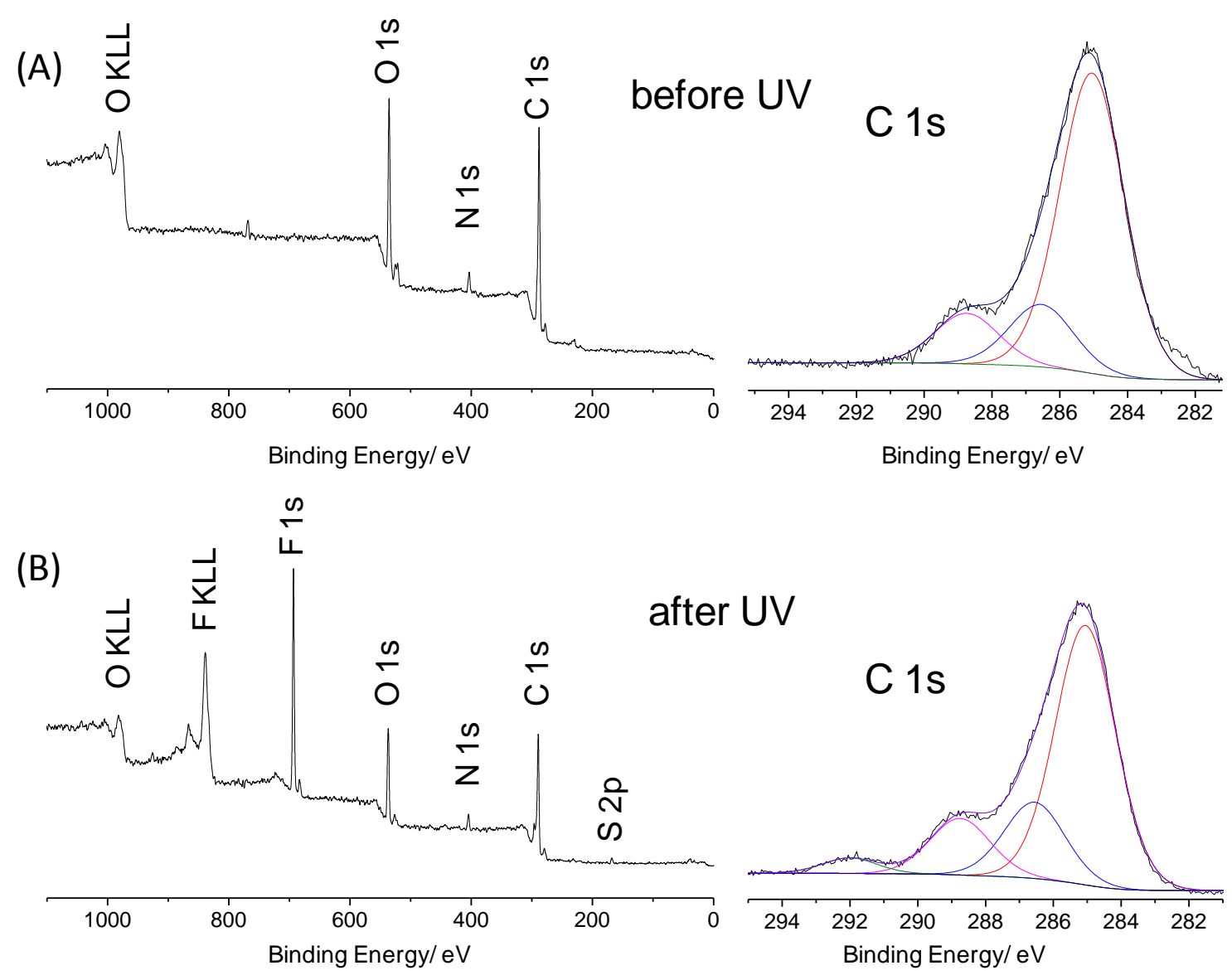

(C)
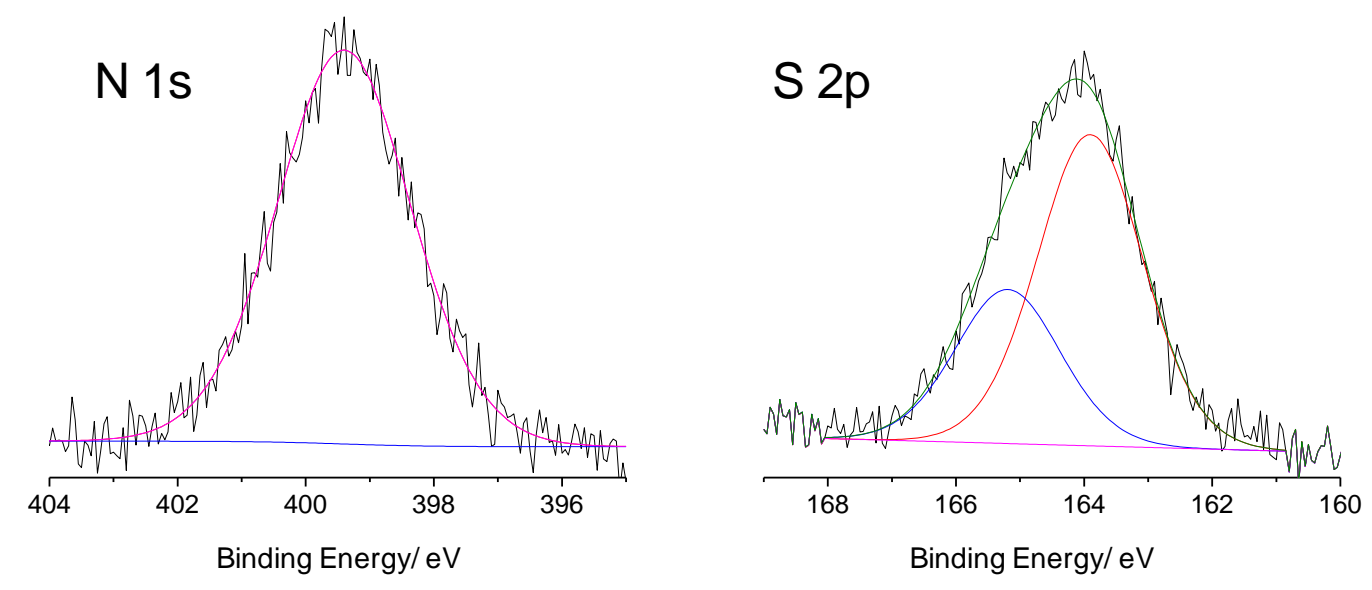
(D)

\begin{tabular}{|c|c|c|}
\hline Irradiation time & Area(N 1s) & Area(S 2p) \\
\hline 2 min & 52425 & 17260 \\
\hline 5 min & 47223 & 19475 \\
\hline $10 \mathrm{~min}$ & 44847 & 20971 \\
\hline $20 \mathrm{~min}$ & 47210 & 23330 \\
\hline $30 \mathrm{~min}$ & 43719 & 21223 \\
\hline
\end{tabular}

(E)

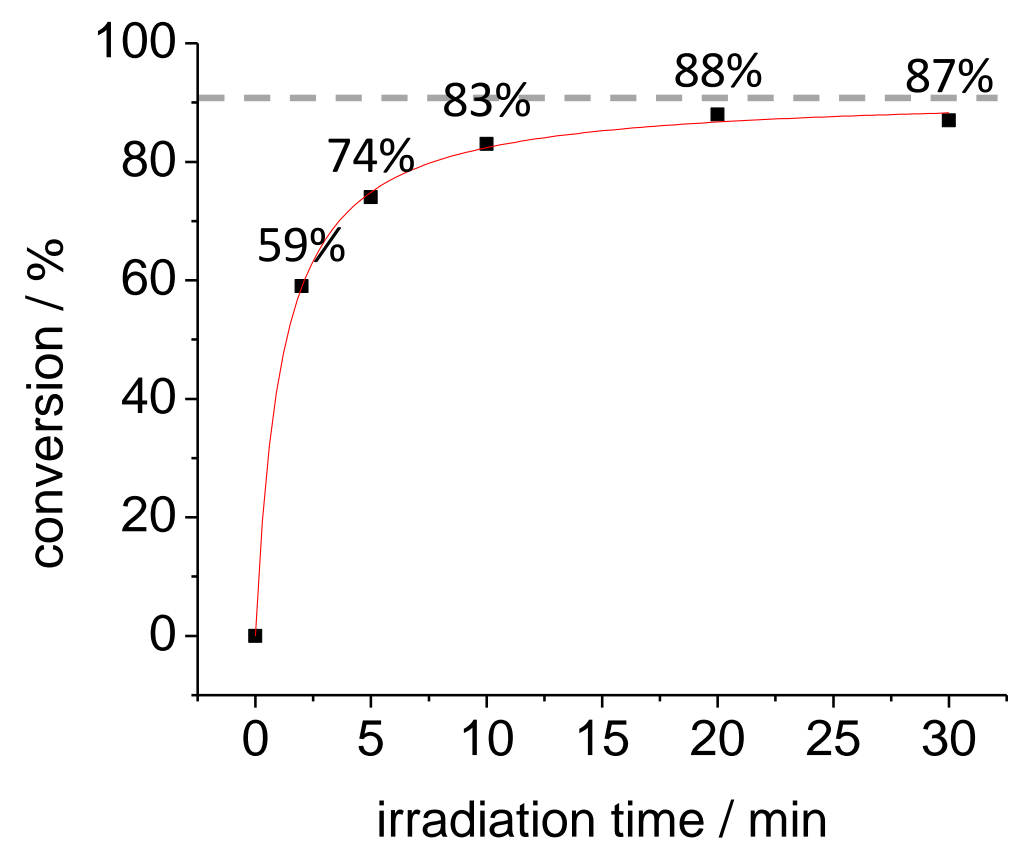

Figure S12. XPS survey spectra and curve-fitted high-resolution XPS C 1s spectra of the unmodified (A) and $1 H, 1 H, 2 H, 2 H$-perfluorodecanethiol-modified (B) tetrazole surface. The new peak with binding energies at $292 \mathrm{eV}$ in $\mathrm{C} 1 \mathrm{~S}$ spectra is the typical characteristics of $-\mathrm{CF}_{3}$ and $-\mathrm{CF}_{2}$ moieties. The main peak in the $\mathrm{C} 1 \mathrm{~S}$ spectrum at $285.0 \mathrm{eV}$ is assigned to saturated carbon atoms $(\mathrm{C}-\mathrm{C}, \mathrm{C}-\mathrm{H})$ and is employed as a reference to compare the evolution of the different species present on the surface. (C) Curve-fitted high-resolution XPS N 1s and S 2p spectra of the thiol 7-modified tetrazole surface. (D) Integration of N1s and S2p peak areas after UV irradiations. (E) Conversions calculated from the integration of N1s and S2p peak areas.

After $1 H, 1 H, 2 H, 2 H$-perfluorodecanethiol 7 modification, 


$$
\text { conversion }=\frac{n(\text { thiol })}{n(\text { tetrazole })} \geq \frac{n(S \text { atom })}{n(N \text { stom }) / 2}=\frac{I(S 2 p) / S(S 2 p)}{I(N 1 s) / 2 S(N 1 s)}
$$

Where $n$ is the number of moles, $I$ is the peak area, and $S$ is the surface sensitivity factor. Here, $S(\mathrm{~S} 2 \mathrm{p})=0.37$ and $S(\mathrm{~N} 1 \mathrm{~s})=0.33^{[5]}$.

The kinetic equation could be established well by using a logistic model.

$$
\begin{gathered}
y=0.91482-0.91473 /\left(1+(t / 1.11724)^{1.00521}\right) \\
R^{2}=0.9980
\end{gathered}
$$

Where $\mathrm{y}$ is the conversion of the surface modification, and $\mathrm{t}$ is the UV irradiation time (min).

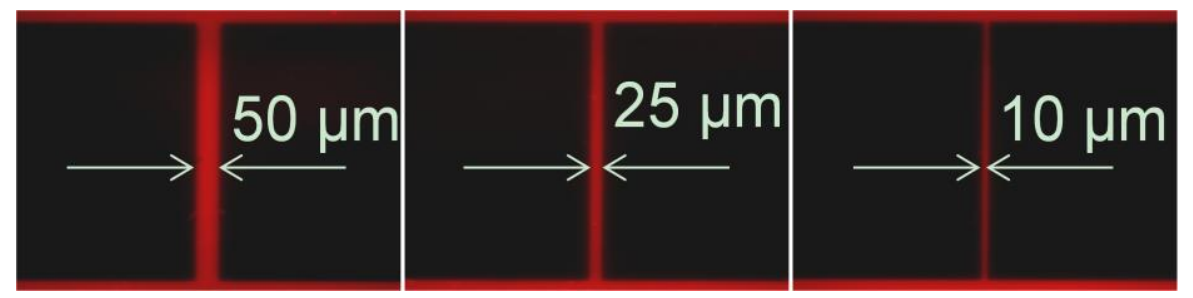

Figure S13. Fluorescence microscope images of Rhodamine-SH pattern on the tetrazole surface with lines of different widths. 


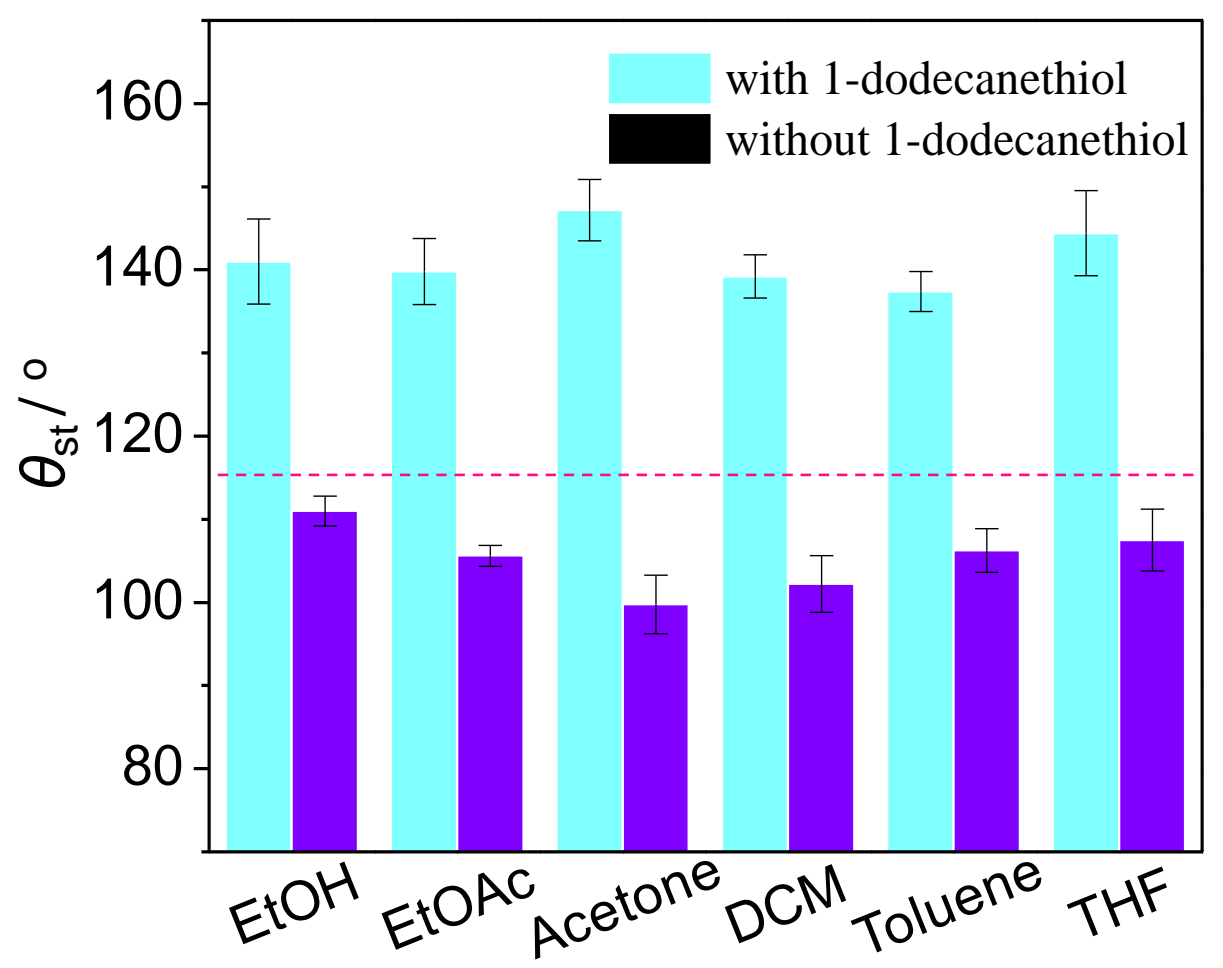

Figure S14. Static water contact angles $\left(\theta_{s t}\right)$ of the tetrazole surfaces functionalized with 1-dodecanethiol dissolved in different solvents via the UV-induced tetrazole-thiol surface modification (cyan). The control experiments were taken under the same conditions without 1-dodecanethiol (violet). Photo modification was carried out for duration of $2 \mathrm{~min}$ with UV irradiation at $260 \mathrm{~nm}$. The dashed pink line means the $\theta_{s t}$ of the original tetrazole surface.

To assess the performance of the UV-induced tetrazole-thiol reaction under different conditions, the tetrazole surface was functionalized by both hydrophobic and hydrophilic thiols dissolved in several broadly used solvents. The tetrazole surfaces $\left(\theta_{\mathrm{st}} 115^{\circ}\right.$ before modification) could be functionalized by $1-$ dodecanethiol in ethanol (EtOH), ethyl acetate (EtOAc), acetone, dichloromethane (DCM), toluene and tetrahydrofuran (THF), which led to an increase of the $\theta_{\text {st }}$ up to $\sim 143^{\circ}$ independently of the solvent used (Figure S13). The grafting of the surface with cysteamine hydrochloride in ethanol transformed the hydrophobic tetrazole surface into hydrophilic $\left(\theta_{\mathrm{st}}=22^{\circ}\right)$. 


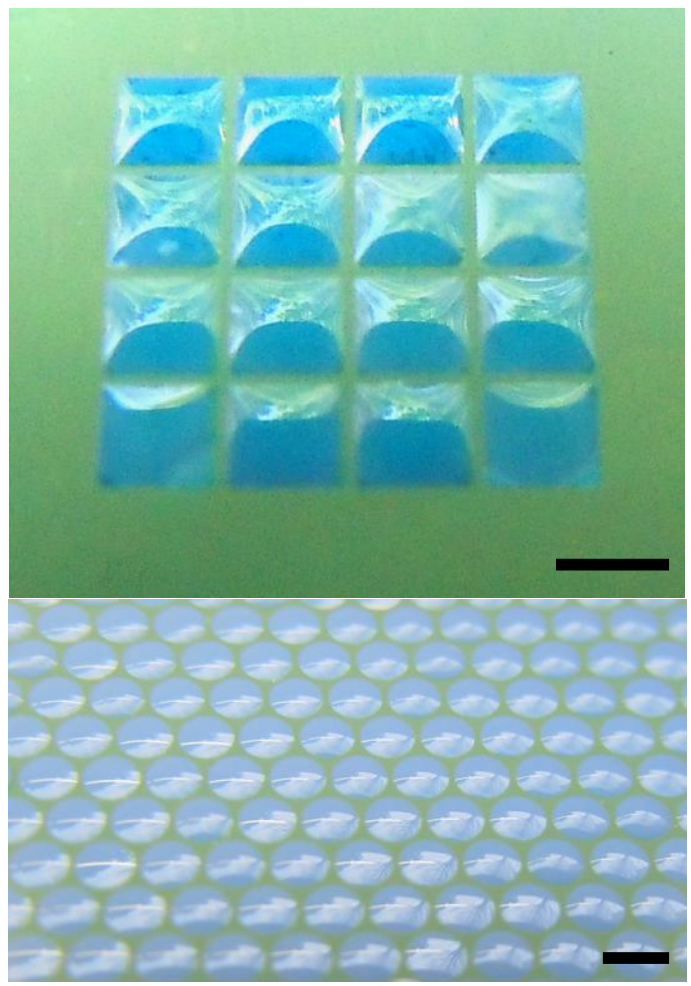

Figure S15. Optical images of superhydrophobic-hydrophilic micropatterns. The hydrophilic spots are filled with dye water solutions. Droplet microarray is formed by the method of discontinuous dewetting ${ }^{[6]}$. Scale bars: $1 \mathrm{~mm}$. 


\section{Materials and Procedures}

2-Hydroxyethyl methacrylate (HEMA) and ethylene dimethacrylate (EDMA) were purchased from SigmaAldrich (Germany) and purified using a short column filled with basic aluminum oxide to eliminate the inhibitors. Biotin-PEG-thiol was purchased from POLYPURE AS, Norway. Alexa Fluor 594 labeled streptavidin was purchased from Thermo Fisher Scientific Inc. All the other chemicals were purchased from Sigma-Aldrich (Germany) and used without further purification. The thiol-containing fluorophore (Rhodamine-SH) was kindly provided by Dr. Junsheng Li. ${ }^{[7]}$ Analytical thin layer chromatography (TLC) was performed on TLC aluminium oxide $60 \mathrm{~F}_{254}$ neutral (Merck) and column chromatography was performed with aluminum oxide neutral (HPLC Flash Grade, 32-63 micron APS Powder, Alfa). Nexterion Glass B UV transparent glass plates (Schott, Germany) were used as substrates for polymer layers. The polymerizations and UV-induced reactions were carried out on an OAI Model 30 deep-UV collimated light source (San Jose, CA) fitted with an USHIO 500 W Hg-xenon lamp (Japan).

\section{Characterization}

SEM images were obtained using the LEO 1530 Gemini scanning electron microscope (Zeiss, Germany) at the Institute of Nanotechnology (INT), KIT. Before SEM measurement, the samples were sputtered with a $30 \mathrm{~nm}$ gold layer using a Cressington 108 auto sputter coater (INT, KIT).

Mass analysis was performed using an electrospray ionization mass spectrometry (ESI-MS) (Bruker ESI-TOF in INT, KIT).

${ }^{1} \mathrm{H}$ spectra were recorded at room temperature using Bruker DRX-500 (500 MHz), and chemical shifts were reported in ppm using residual solvent peaks as internal standards (DMSO-d $\mathrm{d}_{6}, 2.50$ and $\left.\mathrm{CDCl}_{3}, 7.24\right) .{ }^{13} \mathrm{C} \mathrm{NMR}$ spectra were recorded at $125 \mathrm{MHz}$ and chemical shifts were reported in ppm.

UV-Vis absorption spectra were recorded using 1-cm quartz cuvettes on a Varian Cary Eclipse spectrometer. Fluorescence spectra were recorded using 1-cm quartz cuvettes on a Varian Cary Eclipse fluorescence spectrophotometer at room temperature. 
Molecular weight and polydispersity index values of the polymers were obtained by GPC using a Tosoh EcoSEC, TOSOH BIOSCIENCE GmbH (Germany) equipped with an auto sampler and Tosoh EcoSEC RI refractive index detector. THF was used as eluent (flow rate $1 \mathrm{~mL} / \mathrm{min}$ ) at $30{ }^{\circ} \mathrm{C}$. All determinations were performed relative to linear polystyrene standards (Polymer Standard Service, MP 474- 2520000 Da).

The tetrazole surfaces were analyzed with a Leybold-Heraeus MAX200 XPS system using a magnesium anode as the X-ray source.

The distributions of perfluorinated fragments on the surface were confirmed by time of flight secondary ion mass spectrometry (ToF-SIMS) (ION TOF Inc., Münster, Germany), IFG, KIT.

The fluorescence images of the polymer layer after Rhodamine-SH modification were captured using a Leica Confocal Microscope SP5.

A UK 1115 digital camera from EHD imaging (Germany) was used to take images of the water droplet on the surface under ambient conditions. ImageJ software with a Dropsnake plugin was used to measure the water contact angle.

\section{$\underline{\text { Experimental \& Supporting Data }}$}

Preparation of 4-(2-phenyl-2H-tetrazol-5-yl)benzoic acid

The synthesis was carried out following a literature procedure ${ }^{[8]}{ }^{1} \mathrm{H}$ NMR (500

MHz, DMSO-d6) $\delta 13.25(\mathrm{~s}, 1 \mathrm{H}), 8.29(\mathrm{~d}, 2 \mathrm{H}), 8.16(\mathrm{t}, 4 \mathrm{H}), 7.70(\mathrm{t}, 2 \mathrm{H}), 7.63(\mathrm{t}$,

1H); ${ }^{13} \mathrm{C}$ NMR (125 MHz, DMSO-d6) $\delta 166.68,163.78,136.11,132.77,130.38$,

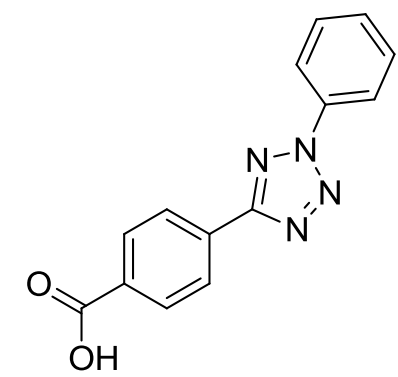

130.27, 130.17, 126.76, 119.95; ESI-MS, calcd. for $\mathrm{C}_{14} \mathrm{H}_{9} \mathrm{~N}_{4} \mathrm{O}_{2} 266.08$ [M-H]', found 265.05. 
Preparation of methyl 4-(2-phenyl-2H-tetrazol-5-yl)benzoate 1

The synthesis was carried out by modifying 4-(2-phenyl-2H-tetrazol-5yl)benzoic acid 2 with methanol via a standard esterification procedure. 4-(2phenyl-2H-tetrazol-5-yl)benzoic acid $(25 \mathrm{mg}, 93 \mu \mathrm{mol})$ was dissolved into 10 $\mathrm{mL}$ of dichloromethane solution containing methanol $(30 \mathrm{mg}, 932 \mu \mathrm{mol})$ and catalyst 4-(dimethylamino)pyridine (DMAP) (56 $\mathrm{mg}, 0.46 \mathrm{mmol})$. Then, the

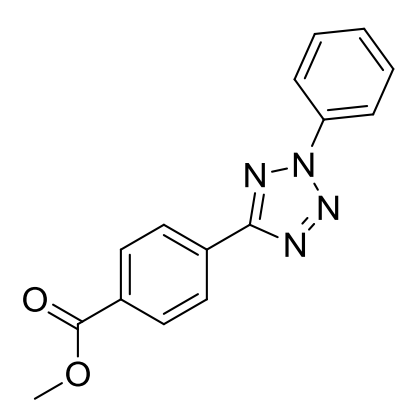
coupling reagent $N, N^{\prime}$-diisopropylcarbodiimide (DIC) $(216 \mu \mathrm{L}, 1.38 \mathrm{mmol})$ was added to the solution at $0^{\circ} \mathrm{C}$. After stirring the solution at RT for $48 \mathrm{~h}$, the reaction mixture was washed with deionized water and the organic layer was collected. The solvent was removed under reduced pressure to give a crude product which was subsequently purified by $\mathrm{Al}_{2} \mathrm{O}_{3}$ column chromatography $\left(\mathrm{CH}_{2} \mathrm{Cl}_{2} /\right.$ hexane $\left.6: 4 \mathrm{v} / \mathrm{v}\right)$ to give the corresponding product. ${ }^{1} \mathrm{H}$ NMR $\left(500 \mathrm{MHz}, \mathrm{CDCl}_{3}\right) 8.33(\mathrm{~d}, 2 \mathrm{H}), 8.19(\mathrm{t}, 4 \mathrm{H}), 7.58(\mathrm{t}, 2 \mathrm{H}), 7.51(\mathrm{t}, 1 \mathrm{H}), 3.95(\mathrm{~s}, 3 \mathrm{H}) ;{ }^{13} \mathrm{C} \mathrm{NMR}$ $\left(125 \mathrm{MHz}, \mathrm{CDCl}_{3}\right) \delta 166.75,164.59,137.02,132.07,131.45,130.45,130.11,129.97,127.2,120.14,52.57$; ESIMS, calcd. for $\mathrm{C}_{15} \mathrm{H}_{13} \mathrm{~N}_{4} \mathrm{O}_{2} 280.10[\mathrm{M}+\mathrm{H}]^{+}$, found 281.11 .

\section{Kinetic study of UV-induced 1,3-dipolar nucleophilic addition with 1 and 2}

Methyl 4-(2-phenyl-2H-tetrazol-5-yl)benzoate 1 and 2-mercaptoethanol 2 were dissolved in ethyl acetate to obtain concentrations of $20 \mu \mathrm{M}$ and $100 \mu \mathrm{M}$, respectively. Separate reactions were set up by adding $2.5 \mathrm{~mL}$ mixture in quartz test tubes. The mixtures were irradiated with a $260 \mathrm{~nm} \mathrm{UV} \mathrm{lamp} \mathrm{(intensity,} 5 \mathrm{~mW} \cdot \mathrm{cm}^{-2}$ ) for 0.5 , $1,2,3,4,5,6,7,8,10 \mathrm{~s}$, respectively. UV-Vis absorption spectra were recorded after irradiation. 
Yield of UV-induced 1,3-dipolar nucleophilic addition with 1 and 2 under different conditions

n(tetrazole $): n($ thiol $)=1: 1$

Methyl 4-(2-phenyl-2H-tetrazol-5-yl)benzoate $1(16.5 \mathrm{mg}, 59 \mu \mathrm{mol})$ and 2-mercaptoethanol $2(4.2 \mu \mathrm{L}, 59 \mu \mathrm{mol})$ were dissolved in $10 \mathrm{~mL}$ ethyl acetate or ethanol in a glass vial. Then the solution was bubbled by argon for 10 min. Under stirring, the solution was irradiated with 260-nm, 312-nm UV lamp, respectively (Intensity, 2 $\mathrm{mW} \cdot \mathrm{cm}^{-2}$ ) for $120 \mathrm{~min}$. After evaporating the solvent under vacuum, the residue was purified by $\mathrm{Al}_{2} \mathrm{O}_{3}$ column chromatography $\left(\mathrm{CH}_{2} \mathrm{Cl}_{2}\right.$ /ethyl acetate $\left.1: 2 \mathrm{v} / \mathrm{v}\right)$ to give the pure product $\mathbf{3}$. The product $\mathbf{3}$ was subsequently weighted and analyzed by MS, ${ }^{1} \mathrm{H}$ NMR and ${ }^{13} \mathrm{C}$ NMR.

n(tetrazole $): \mathrm{n}($ thiol $)=1: 5$

Methyl 4-(2-phenyl-2H-tetrazol-5-yl)benzoate 1 (16.5 mg, $59 \mu$ mol) and 2-mercaptoethanol 2 (21 $\mu \mathrm{L}, 295 \mu \mathrm{mol})$ were dissolved in $10 \mathrm{~mL}$ ethyl acetate or ethanol in a glass vial. Then the solution was bubbled by argon for 10 min. Under stirring, the solution was irradiated with 260-nm, 312-nm and 365-nm UV lamp, respectively (Intensity, $2 \mathrm{~mW} \cdot \mathrm{cm}^{-2}$ ) for $120 \mathrm{~min}$. After evaporating the solvent under vacuum, the residue was purified by $\mathrm{Al}_{2} \mathrm{O}_{3}$ column chromatography $\left(\mathrm{CH}_{2} \mathrm{Cl}_{2} /\right.$ ethyl acetate $\left.1: 2 \mathrm{v} / \mathrm{v}\right)$ to give the pure product 3 . The product 3 was subsequently weighted and analyzed by MS, ${ }^{1} \mathrm{H}$ NMR and ${ }^{13} \mathrm{C}$ NMR.

The yields are shown in Table 1.

To demonstrate that UV-induced tetrazole-thiol reaction can also proceed in water, a tetrazole moiety (4-(2phenyl-2H-tetrazol-5-yl)benzoic acid) was introduced to poly(ethylene glycol)methyl ether (MW=5000 g/mol) (PEG-tetrazole, 4) by esterification, which was confirmed by gel permeation chromatography (GPC) and ${ }^{1} \mathrm{H}$ NMR (Figure S6). Then PEG-tetrazole was used to react with 2-mercaptoethanol (5 eq.) in water under $312 \mathrm{~nm}$ UV light for 3 h. Conversion was estimated based on NMR spectra (Figure S7) after removal of excess thiol. Instead of being quenched by $\mathrm{H}_{2} \mathrm{O}$, the nitrilimine intermediate of PEG-tetrazole 4 reacted with 2-mercaptoethanol 2 , giving rise to the thiohydrazonate product with $95 \%$ conversion. This result proves the practicability of the UVinduced tetrazole-thiol reaction in aqueous solution. 


\section{Preparation of PEG-tetrazole 4}

The synthesis was carried out by modifying poly(ethylene glycol) methyl ether (MW=5000) with 4-(2-phenyl-2Htetrazol-5-yl)benzoic acid via a standard esterification procedure. Poly(ethylene glycol) methyl ether (309.3 mg) was dissolved into $15 \mathrm{~mL}$ of dichloromethane solution containing 4-(2-phenyl-2H-tetrazol-5-yl)benzoic acid (27.3 $\mathrm{mg}, 0.103 \mathrm{mmol}$ ) and catalyst 4-(dimethylamino)pyridine (DMAP) (13 mg, $0.105 \mathrm{mmol})$. Then, the coupling reagent $\mathrm{N}, \mathrm{N}^{\prime}$-diisopropylcarbodiimide (DIC) $(23.2 \mu \mathrm{L}, 0.15 \mathrm{mmol})$ was added to the solution at $0^{\circ} \mathrm{C}$. After stirring the solution at RT for $48 \mathrm{~h}$., $50 \mathrm{~mL}$ of cold diethyl ether was added and the precipitate formed was collected and dried under vacuum. The crude product was re-dissolved in dichloromethane and precipitated by adding cold diethyl ether, this procedure was repeated three times. The corresponding product was characterized by GPC (Figure S6).

\section{UV-induced 1,3-dipolar nucleophilic addition with PEG-tetrazole and 2-mercaptoethanol in water}

PEG-tetrazole $4(71.6 \mathrm{mg})$ and 2-mercaptoethanol $2(6.0 \mu \mathrm{L})$ were dissolved in $6 \mathrm{~mL}$ water in a glass vial. Then the solution was bubbled by argon for 10 min. Under stirring, the solution was irradiated with 312-nm UV lamp (Intensity, $2 \mathrm{~mW} \cdot \mathrm{cm}^{-2}$ ) for $180 \mathrm{~min}$. After evaporating the solvent under vacuum, the residue was redissolved into $2 \mathrm{~mL}$ THF. A fine precipitate was formed by adding $12 \mathrm{~mL}$ diethyl ether and isolated by centrifugation. Repeat

the dissolution and precipitation twice. After drying under vacuum, the solid was analyzed by ${ }^{1} \mathrm{H}$ NMR and ${ }^{13} \mathrm{C}$ NMR to determine the conversion.

\section{Modification of bovine serum albumin (BSA) by PEG-tetrazole}

$25 \mu \mathrm{L}$ of PEG-tetrazole 4 (5 mg/mL in PBS buffer) or PEG (5 mg/mL in PBS buffer) or PBS buffer were added to $25 \mu \mathrm{L}$ solutions of BSA solution ( $3 \mathrm{mg} / \mathrm{mL}$, PBS buffer) in a 96-well microtiter plate. After irradiating with a 312-nm UV lamp for $5 \mathrm{~min}$, the solutions were incubated for $2 \mathrm{~h}$ at $37^{\circ} \mathrm{C}$. The mixtures were boiled at $95^{\circ} \mathrm{C}$ for 5 min after being added $10 \mu \mathrm{L} 5 \times$ SDS sample buffer. The samples were then loaded onto a $8 \%$ polyacrylamide gel 
and subjected to protein electrophoresis at $130 \mathrm{~V}$ for $2 \mathrm{~h}$. The fluorescent bands in the gel were visualized by illuminating the gel under 365-nm UV light. Afterwards, the same gel was stained with Coomassie blue.

\section{Polymer-polymer coupling}

PEG-tetrazole 4 (38 mg) and O-(2-mercaptoethyl)-O'-methylpolyethylene glycol 5 (MW=5000) (36.5 mg) were dissolved in THF ( $25 \mathrm{~mL})$. Separate reactions were set up by adding $2.5 \mathrm{~mL}$ mixture in glass vials. The mixtures were irradiated with a $260 \mathrm{~nm} \mathrm{UV} \mathrm{lamp} \mathrm{(Intensity}=5 \mathrm{~mW} \cdot \mathrm{cm}^{-2}$ ) under argon for $0 \mathrm{sec}, 5 \mathrm{sec}, 15 \mathrm{sec}, 30 \mathrm{sec}, 60$ sec, $4 \mathrm{~min}, 7 \mathrm{~min}$ and $10 \mathrm{~min}$, respectively. Solvents were evaporated under vacuum. The residues were redissolved in THF and withdrawn for GPC measurements.

Figure 2A shows a model polymer conjugation experiment that was conducted. For this purpose, O-(2mercaptoethyl)-O'-methylpolyethylene glycol 5 (MW $5000 \mathrm{~g} / \mathrm{mol}$ ) was utilized as the thiol-terminated polymer (PEG-thiol). Equimolar amounts of 4 and $\mathbf{5}$ were dissolved in THF $(3 \mathrm{mg} / \mathrm{mL})$ and subsequently irradiated with $U V_{\lambda=260 \mathrm{~nm}}$ light. The reaction mixture was analyzed by GPC at different irradiation times (Figure 2B). A distinct shift of the GPC traces to lower elution volumes indicates successful formation of a higher molecular weight polymer 6 by polymer-polymer conjugation. Half of the starting material already reacted after only 1 min of UV irradiation, and no significant difference was observed for samples taken after $7 \mathrm{~min}$. The remaining small GPC peak corresponding to the starting material could be due to the incomplete tetrazole functionalization of the 4 as well as the non- equimolar ratio of the reactants. These results demonstrate that the UV-induced tetrazole-thiol reaction can also be applied for the conjugation of macromolecules.

\section{Preparation of $12.5 \mu \mathrm{m}$-thin porous HEMA-EDMA films}

Here we employed a published procedure developed in our group to make porous HEMA-EDMA polymer layers. ${ }^{[3]}$ Briefly, two $12.5 \mu$ m-thin strips of Teflon film (American Durafilm Co.) were placed at the edges of one 3-(trimethoxysilyl)propyl methacrylate modified glass-plate and one fluorinated glass slide was clamped on top of 
it. $70 \mu \mathrm{L}$ of polymerization mixture of HEMA (24 wt $\%)$, EDMA (16 wt \%), 1-decanol (12 wt \%), cyclohexanol (48 $\mathrm{wt} \%$ ) and 2,2-dimethoxy-2-phenylacetophenone (DMPAP) (photoinitiator, $1 \mathrm{wt} \%$ with respect to monomers) were injected in the mold between the glass slides and irradiated for $15 \mathrm{~min}$ with $5 \mathrm{~mW} \cdot \mathrm{cm}^{-2} 260 \mathrm{~nm}$ UV-light. The mold was then carefully opened using a scalpel. The resulting superficial surface was removed by applying and rapidly removing adhesive film ("Scotch tape") after separating the plates while the layer was still wetted. A homogeneous porous surface was formed. The plate was washed extensively with ethanol and kept in ethanol for some minutes before drying.

\section{Preparation of tetrazole-modified HEMA-EDMA films (tetrazole surface)}

Two glass plates coated with a HEMA-EDMA layer were immersed into $50 \mathrm{~mL}$ of dichloromethane solution containing 4-(2-phenyl-2H-tetrazol-5-yl)benzoic acid (80 mg, $0.3 \mathrm{mmol})$ and catalyst 4-(dimethylamino)pyridine (DMAP) (56 mg, $0.46 \mathrm{mmol})$. Then, the coupling reagent $N, N^{\prime}$-diisopropylcarbodiimide (DIC) (185.5 $\mu \mathrm{L}, 1.2$ mmol) was added to the solution at $0^{\circ} \mathrm{C}$. After stirring the solution at RT for $24 \mathrm{~h}$, the plates were washed extensively with acetone, followed by drying.

\section{UV-induced tetrazole-thiol reactions on tetrazole surface}

$1 H, 1 H, 2 H, 2 H$-perfluorodecanethiol 7 modification: the tetrazole surface was wetted with an ethyl acetate solution containing 20 vol\% $1 \mathrm{H}, 1 \mathrm{H}, 2 \mathrm{H}, 2 \mathrm{H}$-perfluorodecanethiol 7 , covered with a photomask, and irradiated by 5 $\mathrm{mW} \cdot \mathrm{cm}^{-2} 260 \mathrm{~nm}$ UV light for different time. After removing the photomask, the tetrazole surface was washed with acetone extensively and dried with a nitrogen gun.

Rhodamine-SH modification: the tetrazole surface was wetted with an acetone solution containing 1 wt\% Rhodamine-SH, covered with a photomask, and irradiated by $5 \mathrm{~mW} \cdot \mathrm{cm}^{-2} 260 \mathrm{~nm} \mathrm{UV}$ light for $2 \mathrm{~min}$. After removing the photomask, the tetrazole surface was washed with acetone extensively and dried with a nitrogen gun. 1-dodecanethiol modification: 1-dodecanethiol (20 vol\%) was dissolved in ethanol, ethyl acetate, acetone, dichloromethane, toluene, and tetrahydrofuran. The tetrazole surface was wetted with one of these thiol solutions, 
covered with a quartz slide, and irradiated by $5 \mathrm{~mW} \cdot \mathrm{cm}^{-2} 260 \mathrm{~nm}$ UV light for $2 \mathrm{~min}$. After removing the cover, the tetrazole surface was washed with acetone extensively and dried with a nitrogen gun.

Preparation of superhydrophobic-hydrophilic micropatterns via tetrazole-thiol reaction

First, the tetrazole surface was wetted with acetone solution containing 20 vol\% $1 H, 1 H, 2 H, 2 H$ perfluorodecanethiol 7, covered by a photomask, and irradiated by UV light $\left(5 \mathrm{~mW} \cdot \mathrm{cm}^{-2}\right)$ for 2 min. After removing the photomask, the surface was washed with acetone and dried. The polymer layer was wetted with an ethanol-water mixture (1:1) containing $20 \mathrm{wt} \%$ cysteamine hydrochloride and irradiated by UV light for another 2 min. Finally, the plate was washed extensively with acetone and dried with a nitrogen gun. 
Designing a cell microarray on produced superhydrophobic-hydrophilic micropattern

mCherry-expressing rat mammary carcinoma cells were cultured in DMEM containing 10\% of fetal bovine serum (FBS). A cell suspension was obtained by trypsinizing a confluent (80\% monolayer) culture grown in a Petri dish in an incubator $\left(37^{\circ} \mathrm{C}, 5 \% \mathrm{CO}_{2}\right)$ for $2-3$ days. For sterilization, the glass substrate with a superhydrophobic-hydrophilic pattern was kept in ethanol for 20 min, dried in air, and placed in a $10 \mathrm{~mL}$ Petri dish. $5 \mathrm{~mL}$ of cell-suspension was added so that the plate was fully covered (seeding density: 43200 cells $/ \mathrm{cm}^{2}$ ). The culture medium was changed after culturing the seeded array in the incubator for $5 \mathrm{~h}$. The cell array was cultured for another $25 \mathrm{~h}$.

\section{Immobilization of biotin-PEG-thiol on tetrazole surface and Streptavidin binding}

Firstly, the tetrazole surface was wetted by an aqueous solution containing 10 vol\% ethanol. Then the plate was washed extensively with pure water to replace the solution in the pores. Excess water was shaken off from the surface. $2 \mathrm{mg} / \mathrm{mL}$ biotin-PEG-thiol aqueous solution was dropped on the surface. The polymer layer was irradiated with $365-\mathrm{nm} \mathrm{UV} \mathrm{light}\left(2 \mathrm{~mW} \cdot \mathrm{cm}^{-2}\right)$ through a photomask for $10 \mathrm{~min}$. Then, the plate was washed extensively with water. The water wetted surface was then irradiated for another 10 min under 365-nm UV light to decompose the residual tetrazole groups. Substrate surfaces were incubated for $30 \mathrm{~min}$ in PBS buffer at $37{ }^{\circ} \mathrm{C}$. Subsequently, the substrates were covered with a solution of streptavidin (Alexa Fluor 594 labeled streptavidin, $1 \mathrm{mg} / \mathrm{mL}$ ) in PBS buffer. After $30 \mathrm{~min}$, the surfaces were washed with the PBS buffer, rinsed with distilled water and dried with a nitrogen gun. 


\section{Reference:}

[1] ) Y. Wang, W. J. Hu, W. Song, R. K. V. Lim, Q. Lin, Organic Letters 2008, 10, 3725-3728.

[2] R. Huisgen, R. Grashey, M. Seidel, H. Knupfer, R. Schmidt, Justus Liebigs Annalen der Chemie 1962, 658, 169-180.

[3]F. L. Geyer, E. Ueda, U. Liebel, N. Grau, P. A. Levkin, Angew Chem Int Edit 2011, 50, 8424-8427.

[4] W. Feng, L. Li, E. Ueda, J. Li, S. Heißler, A. Welle, O. Trapp, P. A. Levkin, Adv. Mater. Inter. 2014, DOI: 10.1002/admi.201400269.

[5]C. D. Wagner, Journal of Electron Spectroscopy and Related Phenomena 1983, 32, 99-102.

[6] E. Ueda, F. L. Geyer, V. Nedashkivska, P. A. Levkin, Lab on a Chip 2012, 12, 5218-5224.

[7] L. Li, J. Li, X. Du, A. Welle, M. Grunze, O. Trapp, P. A. Levkin, Angew Chem Int Edit 2014, 53, 3835-3839.

[8] W. Song, Y. Wang, J. Qu, M. M. Madden, Q. Lin, Angew Chem Int Edit 2008, 47, 2832-2835. 